$5-2000$

\title{
On the Behavior of a Class of Patched Plates During Cooling
}

Anette M. Karlsson

Cleveland State University, a.karlsson@csuohio.edu

W. J. Bottega

Rutgers University - New Brunswick/Piscataway

Follow this and additional works at: https://engagedscholarship.csuohio.edu/enme_facpub

Part of the Mechanical Engineering Commons

How does access to this work benefit you? Let us know!

Publisher's Statement

NOTICE: this is the author's version of a work that was accepted for publication in International Journal of Non-Linear Mechanics. Changes resulting from the publishing process, such as peer review, editing, corrections, structural formatting, and other quality control mechanisms may not be reflected in this document. Changes may have been made to this work since it was submitted for publication. A definitive version was subsequently published in International Journal of Non-Linear Mechanics, 35, 3, May 2000; 10.1016/S0020-7462(99)00040-2

\section{Original Citation}

Karlsson, A. M., and Bottega, W. J., 2000, "On the Behavior of a Class of Patched Plates during Cooling," International Journal of Non-Linear Mechanics, 35(3) pp. 543-566.

This Article is brought to you for free and open access by the Mechanical Engineering Department at EngagedScholarship@CSU. It has been accepted for inclusion in Mechanical Engineering Faculty Publications by an authorized administrator of EngagedScholarship@CSU. For more information, please contact library.es@csuohio.edu. 


\title{
On the behavior of a class of patched plates during cooling
}

\author{
A.M. Karlsson*, W.J. Bottega \\ Department of Mechanical and Aerospace Engineering, Rutgers University, Piscataway, NJ 08854-8054, USA
}

\section{Introduction}

A configuration occurring in many contemporary engineering structures consists of a primary, or "base", structure to which a secondary component, or "patch", is adhered, forming a "composite structure". Such structures arise in a variety of applications, ranging from thin films on electronic substrates to repair patches on aircraft, and smart structures. A range of selected studies concerned with various aspects of the integrity of patched structures may be found in Refs. [1-10].

An area of concern that is pertinent to the integrity and performance of patched structures is the response of such structures to thermo-mechanical loading conditions. In addition to inducing unwanted residual stresses [11,12], changes in tem-

\footnotetext{
* Corresponding author.
}

perature may induce instability of thin composite structures, either continuously laminated [13-27] or patched [1], resulting in bifurcation buckling, snap-through buckling or simply unacceptably large out-of-plane deflections. Such behavior can evidently have dramatic consequences with regard to the integrity and performance of the composite structure. In this regard, Karlsson and Bottega [1] investigated the response of patched beam-plates during heating. In that study, a non-linear formulation was presented and solved analytically, and numerical simulations based on the corresponding solutions were performed for several loading and support conditions, and revealed a rich and varied behavior of the structures of interest. In that work, a loading parameter was identified, as were two critical parameters, a critical membrane force and a critical temperature. In addition, a stability criterion was established and used to assess the stability of the various equilibrium branches of the 
loading path. Critical behavior observed included bifurcation buckling, "asymptotic buckling", and "sling-shot buckling", with the occurrence and characteristics seen to be a function of the aforementioned critical parameters.

While the response of structures subjected to elevated temperatures is most certainly an important consideration, many structures experience moderate to severe cooling during their service. It is to this issue that the present study is addressed. To this end we consider the response of a class of patched plates during uniform cooling. The plates considered are in either plane strain or plane stress configurations (beam-plates). They may be supported in a variety of ways and may be subjected to applied in-plane edge loads as well. The non-linear formulation of Ref. [1] is adopted and analytical solutions are obtained. In addition, the stability criterion established therein is extended to the present case, and numerical simulations based on the analytical solutions are performed revealing a rich behavior and the parameters that control such behavior. A detailed discussion of the intricate results of a variety of cases provides insight into the rather complex thermo-mechanical behavior of structures of the class considered when subjected to the thermal conditions of interest, and identifies the factors that govern such behavior.

\section{Problem statement}

In the present study we are concerned with the behavior of patched plates in plane strain or plane stress configurations, during uniform cooling from some reference temperature. To this end, we adopt the formulation presented in Ref. [1], using the same notation and direct reference to that study where appropriate.

Consider a thin flat structure comprised of a base plate of normalized half-span $L \equiv 1$ to which a patch of half-span $L_{\mathrm{p}} \leqslant 1$ is perfectly adhered. The region where the patch is present is defined as $S_{1}: x \in\left[0, L_{\mathrm{p}}\right]$, as shown in Fig. 1 . The coordinate $x$ runs along the upper surface of the base plate and originates at the centerspan of the structure, as shown. Furthermore, the region ahead of the patch (i.e., the region of the composite structure that

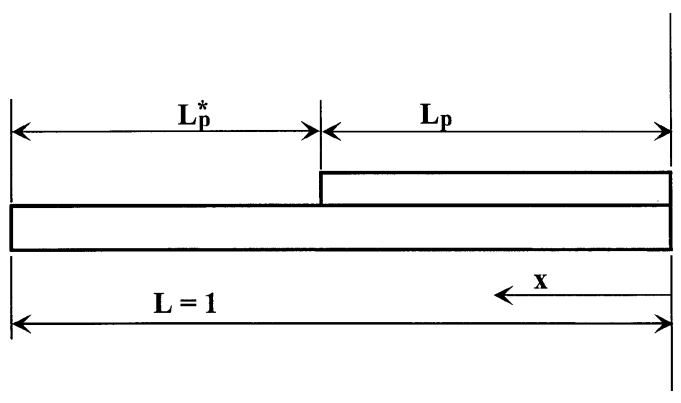

Fig. 1. Geometry of patched plate.

consists only of the base structure), is defined as $S_{2}: x \in\left[L_{\mathrm{p}}, 1\right]$. We shall be interested in examining the response of the "composite structure" comprised of the patch and base plate when it is subjected to a uniform temperature change below some reference temperature. In what follows all length scales are normalized with respect to the dimensional half-span $\bar{L}$ of the undeformed structure and the temperature change, $\Theta<0$, is normalized with respect to the reference temperature. The interface between the patch and base plate, and its extension (i.e., the upper surface of the base plate), is used as the reference surface. The base plate and the patch are modeled individually as von Karman plates, with the composite structure considered as an assemblage of the two primitive structures. To this end, the in-plane and transverse displacements and the corresponding strain and curvature at the centerline of the base plate within region $S_{i}(i=1,2)$ are designated as $u_{i}(x)$ (positive in direction of increasing $x$ ) and $w_{i}(x)$ (positive downward), and $e_{i}(x)$ and $\kappa_{i}(x)$, respectively. The corresponding measures for the patch, at its centerline, within region $S_{1}$ are designated as $u_{\mathrm{p}}(x), w_{\mathrm{p}}(x), e_{\mathrm{p}}(x)$ and $\kappa_{\mathrm{p}}(x)$, in the same sense. The respective measures at the reference surface are indicated by an asterisk, i.e. $u_{i}^{*}(x), w_{i}^{*}(x), e_{i}^{*}(x), \kappa_{i}^{*}(x)$, and $u_{\mathrm{p}}^{*}(x), w_{\mathrm{p}}^{*}(x), e_{\mathrm{p}}^{*}(x)$, $\kappa_{\mathfrak{p}}^{*}(x)$. The strain-displacement and curvaturedisplacement relations, along with the associated through the thickness variations, for the particular plate theory employed may be found in Appendix A.

The self-consistent set of governing differential equations, boundary and matching conditions 
derived in Ref. [1] are given below. ${ }^{1}$ The differential equations are

$$
\begin{aligned}
& M_{1}^{* \prime \prime}-\left(N_{1}^{*} w_{1}^{* \prime}\right)^{\prime}=0 \quad\left(x \in S_{1}\right), \\
& N_{1}^{* \prime}=0 \quad\left(x \in S_{1}\right), \\
& M_{2}^{\prime \prime}-\left(N_{2} w_{2}^{\prime}\right)^{\prime}=0 \quad\left(x \in S_{2}\right), \\
& N_{2}^{\prime}=0 \quad\left(x \in S_{2}\right),
\end{aligned}
$$

with

$$
\begin{array}{ll}
w_{1}^{*}(x) \equiv w_{1}(x) & \left(x \in S_{1}\right), \\
w_{1}(x)=w_{\mathrm{p}}(x) & \left(x \in S_{1}\right), \\
\kappa_{1}^{*}(x) \equiv \kappa_{1}(x) & \left(x \in S_{1}\right), \\
\kappa_{1}(x)=\kappa_{\mathrm{p}}(x) & \left(x \in S_{1}\right), \\
u_{1}^{*}(x)=u_{\mathrm{p}}^{*}(x) & \left(x \in S_{1}\right),
\end{array}
$$

where

$$
\begin{aligned}
N_{1}^{*}(x) & =C^{*} e_{1}^{*}(x)+B^{*} \kappa_{1}^{*}(x)-n^{*} \Theta \\
& =C^{*}\left[e_{1}^{*}(x)-\alpha^{*} \Theta\right]+B^{*}\left[\kappa_{1}^{*}(x)-\beta^{*} \Theta\right], \\
M_{1}^{*}(x) & =A^{*} \kappa_{1}^{*}(x)+B^{*} e_{1}^{*}(x)-\mu^{*} \Theta \\
& =A^{*}\left[\kappa_{1}^{*}(x)-\beta^{*} \Theta\right]+B^{*}\left[e_{1}^{*}(x)-\alpha^{*} \Theta\right] \\
& =D^{*}\left[\kappa_{1}^{*}(x)-\beta^{*} \Theta\right]+\rho^{*} N_{1}^{*},
\end{aligned}
$$

respectively, correspond to the normalized membrane force and normalized bending moment in the patched portion of the composite structure,

$N_{2}(x)=C\left[e_{2}(x)-\alpha \Theta\right]$,

$M_{2}(x)=D \kappa_{2}(x)-\frac{1}{2} h N_{2}$,

correspond to the normalized membrane force and normalized bending moment in the base structure

\footnotetext{
${ }^{1}$ In the variational formulation of Ref. [1] the thermomechanical strain energies are included in an energy functional together with a constraint functional that matches the displacement of the primitive structures at the bondline in the patched region. The principal of stationary potential energy is then invoked resulting in the governing equations and conditions.
}

outside the patched region and superposed primes denote differentiation with respect to $x$. The normalized stiffnesses and thermal coefficients, $C, D, \alpha$, $C_{\mathrm{p}}, D_{\mathrm{p}}, \alpha_{\mathrm{p}}$, of the primitive structures, as well as those corresponding to the intact segment of the composite structure, $A^{*}, B^{*}, C^{*}, D^{*}, \rho^{*}, \alpha^{*}, \beta^{*}$, are given in Appendix B.

The associated boundary and matching conditions are given by

\section{Symmetric deformation}

$u_{1}^{*}(0)=0$,

$w_{1}^{*^{\prime}}(0)=0$,

$\left[M_{1}^{* \prime}-N_{1}^{*} w_{1}^{* \prime}\right]_{x=0}=0$

or

antisymmetric deformation

$\hat{u}(0)=\hat{\rho} w_{1}^{*^{\prime}}(0)$,

$D^{*} \kappa_{1}^{*}(0)=0$,

$w_{1}^{*}(0)=0$,

and for both cases

$u_{1}^{*}\left(L_{\mathrm{p}}\right)=u_{2}^{*}\left(L_{\mathrm{p}}\right)$,

$N_{1}^{*}\left(L_{\mathrm{p}}\right)=N_{2}\left(L_{\mathrm{p}}\right)$,

$w_{1}^{*}\left(L_{\mathrm{p}}\right)=w_{2}\left(L_{\mathrm{p}}\right)$,

$w_{1}^{* \prime}\left(L_{\mathrm{p}}\right)=w_{2}^{\prime}\left(L_{\mathrm{p}}\right)$,

$M_{1}^{*}\left(L_{\mathrm{p}}\right)=M_{2}\left(L_{\mathrm{p}}\right)$,

$\left[M_{1}^{* \prime}-N_{1}^{*} w_{1}^{* \prime}\right]_{x=L_{\mathrm{p}}}=\left[M_{2}^{\prime}-N_{2} w_{2}^{\prime}\right]_{x=L_{\mathrm{p}}}$,

$u_{2}(1)=0$

or

$N_{2}(1)=T_{0}\left(T_{0}\right.$ prescribed $)$,

and

$w_{2}(1)=0$,

and

$w_{2}^{\prime}(1)=0$ 
or

$\kappa_{2}(1)=0$,

where

$\hat{u}_{1}(x)=u_{1}^{*}(x)+\rho^{*} w_{1}^{* \prime}(x)$

is the in-plane deflection of the neutral surface of the composite structure in the patched region, and

$\hat{\rho} \equiv m^{*} \Theta / N_{1}^{*}$

locates the transverse distance from the centroidal plane to the "effective" neutral plane (i.e., the plane with vanishing moment).

Integration of Eqs. (1b) and (2b), and imposition of the associated matching condition ( $7 \mathrm{~b}$ ) yields the results

$N_{1}^{*}=N_{2}=$ constant $=N_{0}$,

where $N_{0}$ is a (yet to be determined) membrane force, for which positive values $\left(N_{0}>0\right)$ correspond to tension. ${ }^{2}$

Finally, integrating the strain-displacement relations and imposing the corresponding boundary and matching conditions for the in-plane displacements results in the integrability condition given by

$$
\begin{aligned}
u_{2}(L)-\hat{u}_{1}(0)= & N_{0}\left[\frac{L_{\mathrm{p}}^{*}}{C}+\frac{L_{\mathrm{p}}}{C^{*}}\right]+\left[L_{\mathrm{p}}^{*} \alpha+L_{\mathrm{p}} \alpha_{1}\right] \Theta \\
& -\left[\rho^{*}+\frac{h}{2}\right] w^{\prime}\left(L_{\mathrm{p}}\right)-\sum_{i=1}^{2} \int_{s_{i}} \frac{1}{2} w_{i}^{\prime 2} \mathrm{~d} x
\end{aligned}
$$

where $\hat{u}_{1}(x)$ is defined by Eq. (9a) and $L_{\mathrm{p}}^{*} \equiv 1-L_{\mathrm{p}}$ corresponds to the (half) length of the unpatched segment of the base plate.

The counterparts of Eqs. (1a) and (2a) and the corresponding boundary and matching conditions obtained upon substitution of the result given by Eq. (10), together with the integrability condition (11), transform the problem statement into a mixed formulation in terms of the transverse displacement

\footnotetext{
${ }^{2}$ In Ref. [1] $N_{0}>0$ corresponds to a compressive membrane force.
}

$w(x)$, the membrane force $N_{0}$, and the temperature (change) $\Theta$.

The loading parameter for the problem is denoted by $M_{\lambda}$ and is defined by the following. Substituting the expressions for the moments in regions 1 and 2, given by Eqs. (4b) and (5b), into the matching condition for the moments over the end of the patch, Eq. (7e), and incorporating Eq. (10), we find that the condition in question takes the form

$$
\left[D^{*} \kappa_{1}^{*}-D \kappa_{2}\right]{ }_{\mathrm{p}}=\mathscr{M}_{\lambda},
$$

where

$\mathscr{M}_{\lambda} \equiv m^{*} \Theta-\left(\rho^{*}+\frac{1}{2} h\right) N_{0}$.

Upon consideration of the governing differential equations and the remaining boundary and matching conditions augmented by the result (10), it may be seen that the loading parameter $\mathscr{M}_{\lambda}$, and hence the temperature, appears only at the matching of the moments at the edge of the patch. In this context $\mathscr{M}_{\lambda}$ may be interpreted as a moment that is applied at $x=L_{\mathrm{p}}$, due to the mismatch in coefficients of thermal expansion between the patch and the base structure and due to the jump of the neutral surface.

The analytical solutions to the non-linear problems of interest for several support and loading conditions are discussed in the next section.

\section{Analytical solution}

In this section we present analytical solutions for the problems stated in Section 2, for selected boundary and loading conditions. As the geometry and material properties of the system are symmetric about the center of its span, we will first be concerned with symmetric solutions. However, we shall also consider the possibility of antisymmetric solutions.

\subsection{Symmetric solutions}

In this subsection we present the analytical solution for the non-linear problem presented in Section 2, assuming symmetric deformation. In the case of non-vanishing membrane force, the general 
solution for the current problem is delineated according to the type of rotational support conditions and is given below.

Hinged end conditions:

$$
\begin{aligned}
w_{1}^{*}(x)= & \frac{\mathscr{M}_{\lambda}}{N_{0} \mathscr{H}_{(\mathrm{h})}} \\
& \times\left[\mathscr{H}_{(\mathrm{h})}+\mathscr{A}_{1} \cosh \left(k^{*} x\right)\right] \quad\left(0 \leqslant x \leqslant L_{\mathrm{p}}\right),
\end{aligned}
$$

$$
\begin{aligned}
w_{2}(x)= & -\frac{\mathscr{M}_{\lambda}}{N_{0} \mathscr{H}_{(\mathrm{h})}} \\
& \times \mathscr{B}_{0} \sinh (k(1-x)) \quad\left(L_{\mathrm{p}} \leqslant x \leqslant 1\right),
\end{aligned}
$$

where

$$
\begin{aligned}
\mathscr{H}_{(\mathrm{h})}= & \mathscr{H}_{(\mathrm{h})}\left(N_{0} ; \mathbf{S}\right) \\
= & -\sqrt{\frac{D^{*}}{D}} \cosh \left(k^{*} L_{\mathrm{p}}\right) \cosh \left(k L_{\mathrm{p}}^{*}\right) \\
& -\sinh \left(k^{*} L_{\mathrm{p}}\right) \sinh \left(k L_{\mathrm{p}}^{*}\right) .
\end{aligned}
$$

\section{Clamped end conditions:}

$w_{1}^{*}(x)$

$$
\begin{aligned}
= & \frac{\mathscr{M}_{\lambda}}{N_{0} \mathscr{H}_{(\mathrm{c})}} \\
& \times\left[\mathscr{H}_{(\mathrm{c})}+\mathscr{A}_{2} \cosh \left(k^{*} x\right)-\mathscr{B}_{0}\right] \quad\left(0 \leqslant x \leqslant L_{\mathrm{p}}\right),
\end{aligned}
$$

$w_{2}(x)$

$$
\begin{aligned}
= & -\frac{\mathscr{M}_{\lambda}}{N_{0} \mathscr{H}_{(\mathrm{c})}} \\
& \times \mathscr{B}_{0}[1-\cosh (k(1-x))] \quad\left(L_{\mathrm{p}} \leqslant x \leqslant 1\right),
\end{aligned}
$$

where

$$
\begin{aligned}
\mathscr{H}_{(\mathrm{c})}=\mathscr{H}_{(\mathrm{c})}\left(N_{0} ; \mathbf{S}\right)= & \sinh \left(k^{*} L_{\mathrm{p}}\right) \cosh \left(k L_{\mathrm{p}}^{*}\right) \\
& +\sqrt{\frac{D^{*}}{D}} \cosh \left(k^{*} L_{\mathrm{p}}\right) \sinh \left(k L_{\mathrm{p}}^{*}\right),
\end{aligned}
$$

with

$\mathscr{A}_{1}=\sqrt{\frac{D^{*}}{D}} \cosh \left(k L_{\mathrm{p}}^{*}\right)$,

$\mathscr{A}_{2}=-\sqrt{\frac{D^{*}}{D}} \sinh \left(k L_{\mathrm{p}}^{*}\right)$,

$\mathscr{B}_{0}=\sinh \left(k^{*} L_{\mathrm{p}}\right)$,

$k^{* 2}=N_{0} / D^{*}$

and

$k^{2}=N_{0} / D$

In Eqs. (15) and (17), $\mathbf{S}$ represents the set of stiffnesses of the structure, and we recall that $L_{\mathrm{p}}^{*}=$ $1-L_{\mathrm{p}}$. The general solution given in Eqs. (14a), (14b), (16a) and (16b) is valid for $N_{0} \neq 0$ throughout the structure. The solution corresponding to the case of vanishing membrane force, $N_{0}=0$, is given in Ref. [1] and thus is not repeated herein.

If, for any of the cases considered above, Eqs. (14a), (14b), (16a) and (16b), the edges of the base plate are free to translate in the plane, the corresponding in-plane edge deflection may be found upon substitution of the appropriate solution into the integrability condition (11). For the case where the edges are fixed with regard to in-plane motion, Eq. (11) will give the non-trivial relationship between the membrane force and the temperature for each of the solutions presented above. For the latter case, the integrability condition may be solved numerically for the membrane force $N_{0}$, for a given temperature $\Theta$. Since we are here concerned with negative temperatures, it may be expected that $N_{0}>0$ for situations where the edges are fixed with regard to in-plane motion.

Eqs. (14a), (14b), (16a) and (16b) possess three important quantities, $\mathscr{M}_{\lambda}, \mathscr{H}_{(\mathrm{h})}\left(N_{0} ; \mathbf{S}\right), \quad$ and $\mathscr{H}_{\text {(c) }}\left(N_{0} ; \mathbf{S}\right)$, where the former is defined in Eq. (13) and the latter two in Eqs. (15) and (17), respectively. The significance of these quantities was discussed in Ref. [1] and are summarized here for clarity. For ease of presentation, we drop the subscripts associated with the particular type of rotational edge 
condition and refer to a generic $\mathscr{H}\left(N_{0} ; \mathbf{S}\right)$ in the following discussion. It may be seen from Eqs. (14a), (14b), (16a) and (16b) that if the pertinent function $\mathscr{H}\left(N_{0} ; \mathbf{S}\right)$ approaches zero, the deflections become large, and that when $\mathscr{H}\left(N_{0} ; \mathbf{S}\right)$ vanishes the deformation becomes singular (i.e., is undefined). It can be easily verified that $\mathscr{H}\left(N_{0} ; \mathbf{S}\right)$ only vanishes for compressive membrane forces (i.e., when $\left.N_{0}<0\right){ }^{3}$ It will be seen that the compressive membrane force associated with the singular case is associated with a bifurcation of the loading path for the case of force controlled edge loading (free inplane edge conditions). The equation

$\mathscr{H}\left(N_{0}, \mathbf{S}\right)=0$

may therefore be interpreted as the associated "characteristic equation". A membrane force satisfying the characteristic equation will be referred to as a "critical membrane force", and will therefore be denoted as $N_{\mathrm{cr}}\left(N_{\mathrm{cr}}<0\right)$. There is evidently more than one such membrane force for a given structure. It may be seen from Eqs. (15) and (17) that $N_{\mathrm{r}}$ is independent of the temperature and the coefficients of thermal expansion.

As was anticipated in Section 2, solutions (14)-(18) are seen to be proportional to the parameter $\mathscr{M}_{\lambda}$, where $\mathscr{M}_{\lambda}$, as defined by Eq. (13), is proportional to $N_{0}$ and $\Theta$. It is further seen from these solutions that vanishing load parameter, $\mathscr{M}_{\lambda}=0$, is associated with vanishing of the transverse displacement over the entire span of the structure. Hence, the vanishing of the loading parameter is associated with flat configurations of the deforming structure. However, it may be seen from Eq. (13) that $\mathscr{M}_{\lambda}$ vanishes for an appropriate ratio of $N_{0}$ and $\Theta$ provided that the sign of $m^{*} \Theta$ and the sign of $N_{0}$ are the same for a given configuration. Since we are concerned with negative temperature changes, a structure loaded with a tensile

\footnotetext{
${ }^{3}$ This follows from the fact that the trigonometric functions $\cosh (s)$ and $\sinh (s)$ are monotonically increasing for increasing characteristic argument $s$, if $s$ is real. If the argument $s$ is imaginary, $s=\mathrm{i} \hat{s}$ where $\mathrm{i}=\sqrt{-1}$, it follows that $\cosh (\mathrm{i} \hat{s})=\cos (\hat{s})$, and $\sinh (\mathrm{i} \hat{\mathrm{s}})=\mathrm{i} \sin (\hat{s})$. For the case under consideration $k$ and $k^{*}$ will be imaginary for a compressive force (see Ref. [1]).
}

membrane force $\left(N_{0}>0\right)$ can only possess a flat configuration if $m^{*} \Theta>0$ (except, of course, for the trivial case where $\Theta=N_{0}=0$ ). Conversely, for a structure subjected to a compressive in-plane edge load, a flat configuration may be achieved only if $m^{*} \Theta<0$. Therefore, if a structure is supported in such a manner that the edges of the base plate are free to allow in-plane motion, a loading program may be constructed in such a way that the structure remains flat throughout the loading sequence. (Alternatively, such a structure may be subjected to temperature controlled loading with $N_{0}$ fixed, or $N_{0}$ controlled loading with $\Theta$ fixed, with a flat configuration eventually being realized when the critical ratio of $\Theta$ and $N_{0}$ is achieved.) For the situations where the edges of the base plate are fixed with regard to in-plane motion, the membrane force and temperature cannot be prescribed independently [1].

For the special case when $\mathscr{M}_{\lambda}$ indeed vanishes, we may solve for the corresponding ratio of temperature and membrane force using Eq. (13). For the particular case where $N_{0}=N_{\mathrm{cr}}$, we define the corresponding "critical temperature" $\Theta=\Theta_{\text {cr }}$, as

$\Theta_{\mathrm{r}} \equiv \frac{\rho^{*}+\frac{1}{2} h}{m^{*}} N_{\mathrm{r}}$.

Since we are presently concerned with negative temperature (changes) and since $N_{\mathrm{cr}}<0$, it may be seen that a negative critical temperature may only exist for cases where $m^{*}>0 .{ }^{4}$ It will be seen that the critical temperature is closely associated with the characterization of the structural response of the composite system.

Finally, we consider the case where both the loading parameter, $\mathscr{M}_{\lambda}$, and the characteristic function, $\mathscr{H}\left(N_{0} ; \mathbf{S}\right)$, vanish simultaneously. The solution for the transverse deflection for this case is

Hinged end conditions:

$$
\begin{array}{ll}
w_{1}^{*}(x)=A_{0}\left(\mathscr{A}_{1} / \mathscr{B}_{0}\right) \cosh \left(k^{*} x\right) & \left(0 \leqslant x \leqslant L_{\mathrm{p}}\right), \\
w_{2}(x)=-A_{0} \sinh (k(1-x)) & \left(L_{\mathrm{p}} \leqslant x \leqslant 1\right) .
\end{array}
$$

\footnotetext{
${ }^{4}$ Positive critical temperature may only exist for $m *<0[1]$.
} 
Clamped end conditions:

$w_{1}^{*}(x)$

$$
=A_{0}\left[-1+\left(\mathscr{A}_{2} / \mathscr{B}_{0}\right) \cosh \left(k^{*} x\right)\right] \quad\left(0 \leqslant x \leqslant L_{\mathrm{p}}\right),
$$

$w_{2}(x)$

$$
=-A_{0}[1-\cosh (k(1-x))] \quad\left(L_{\mathrm{p}} \leqslant x \leqslant 1\right),
$$

where $A_{0}$ is an arbitrary constant and $\mathscr{A}_{1}, \mathscr{A}_{2}, \mathscr{B}_{0}, k$, and $k^{*}$ are given by Eqs. (18a), (18b), (18c), (18d) and (18e), respectively. For the present case of cooling temperatures, it may be seen that simultaneous vanishing of $\mathscr{M}_{\lambda}$ and $\mathscr{H}$ corresponds to bifurcation buckling if the edges are free to allow in-plane motion. However, in the case of the edges being fixed from in-plane motion, a tensile membrane force will result upon cooling. In this case $\mathscr{H}\left(N_{0} ; \mathbf{S}\right)$ may not vanish, since it is required that $N_{0}<0$ for the characteristic Eq. (19) to be satisfied. Thus, for negative temperature changes, the simultaneous vanishing of the loading parameter, $\mathscr{M}_{\lambda}$, and the characteristic function, $\mathscr{H}\left(N_{0} ; \mathbf{S}\right)$, may only occur for the case where the edges are free to allow in-plane motion.

We next examine the existence of antisymmetric solutions for the problem of interest.

\subsection{Existence of antisymmetric solutions}

It was seen in Ref. [1] that a structure that is symmetrically deformed may buckle in an antisymmetric manner, once a corresponding critical load is reached, but may not generally do so otherwise. Paralleling the discussion in Ref. [1] it is found that these conclusions are valid for the present case of decreasing temperature.

In this regard, the critical load for antisymmetric deformations $N_{\mathrm{r}_{\mathrm{AS}}}$ may be found from the corresponding "characteristic equation",

$\mathscr{H}_{\mathrm{AS}}\left(N_{0} ; \mathbf{S}\right)=0$,

where $\mathscr{H}_{\mathrm{AS}}\left(N_{0} ; \mathbf{S}\right)$ is dependent on the support conditions as follows:
Hinged end conditions:

$$
\begin{aligned}
\mathscr{H}_{\mathrm{AS}}=\mathscr{H}_{(\mathrm{h})}\left(N_{0} ; \mathbf{S}\right)= & \sinh \left(k^{*} L_{\mathrm{p}}\right) \cosh \left(k L_{\mathrm{p}}^{*}\right) \\
& +\sqrt{\frac{D}{D^{*}}} \cosh \left(k^{*} L_{\mathrm{p}}\right) \sinh \left(k L_{\mathrm{p}}^{*}\right) .
\end{aligned}
$$

Clamped end conditions:

$$
\begin{aligned}
\mathscr{H}_{\mathrm{AS}}=\mathscr{H}_{(}\left(N_{0} ; \mathbf{S}\right)= & {\left[\sinh \left(k L_{\mathrm{p}}^{*}\right)-k \cosh \left(k L_{\mathrm{p}}^{*}\right)\right] } \\
& \times \sqrt{\frac{D}{D^{*}}} \cosh \left(k^{*} L_{\mathrm{p}}\right) \\
& +\left[-k \sinh \left(k L_{\mathrm{p}}^{*}\right)\right. \\
& \left.+\cosh \left(k L_{\mathrm{p}}^{*}\right)\right] \sinh \left(k^{*} L_{\mathrm{p}}\right) .
\end{aligned}
$$

Since multiple solutions (i.e., multiple equilibrium configurations for a given value of the loading parameter) may result due to the non-linearity of the problem, stability of the equilibrium configurations must be addressed. This is done in the next section.

\section{Stability criterion}

When multiple equilibrium configurations of the structure are possible for a given value of the loading parameter, it is of interest to determine which of the configurations are stable and which are unstable. In this regard, stability will be assessed by examination of the second variation of the potential energy of the system. A particular equilibrium configuration will be considered stable if the second variation of the total energy is positive definite for that state, in the context of perturbations away from it (see, for example, Ref. [28]).

Paralleling the development in Ref. [1], which involves perturbations in the form of small moments applied at the edge of the patch $\left(\delta \mathscr{M}_{\lambda}\right)$, the stability criterion for the present case may be stated as follows:

An equilibrium configuration is stable if $\mathscr{F} / N_{0}>0$, and unstable otherwise. 
where

$\mathscr{F}=\frac{1}{4}\left(f_{\mathscr{A}}^{2} k^{*} \sinh 2 k^{*} L_{\mathrm{p}}+f_{\mathscr{B}}^{2} k \sinh 2 k L_{\mathrm{p}}^{*}\right)$,

and

$f_{\mathscr{A}}=\mathscr{A}_{1} / \mathscr{H}_{(\mathrm{h})} \quad$ (hinged end conditions),

$f_{\mathscr{B}}=\mathscr{B}_{0} / \mathscr{H}_{(\mathrm{h})} \quad$ (hinged end conditions),

$\left.f_{\mathscr{A}}=\mathscr{A}_{2} / \mathscr{H}_{(}\right)$(clamped end conditions),

$\left.f_{\mathscr{B}}=\mathscr{B}_{0} / \mathscr{H}_{(}\right)$(clamped end conditions).

$\mathscr{A}_{1}, \mathscr{A}_{2}$, and $\mathscr{B}_{0}$ are defined by Eqs. (18a), (18b) and (18c), and $\mathscr{H}_{(\mathrm{h})}$ and $\mathscr{H}_{(\text {) }}$ are defined by Eqs. (15) and (17), respectively.

With the analytical solutions and stability criterion established, we next present results of numerical simulations, which elucidate the structural behavior of the patched plate.

\section{Results and discussion}

In this section, results are presented for patched plates subjected to a uniform applied temperature field, $\Theta<0$, under various loading scenarios and support conditions. With regard to the latter, we consider plates where the edges are either hinged or clamped with regard to rotations and are either free or fixed with regard to in-plane translation. In each case the analytical solutions based on the nonlinear formulation presented earlier are employed. For the case where the edges are free to allow in-plane motion, we consider an in-plane force applied at the edge of the structure and examine the behavior of the composite structure for a range of loading situations. For the case where the edges are fixed so as to prohibit in-plane motion we examine the temperature controlled behavior of the composite structure. The scenario for the former will be discussed in Section 5.1 and that for the latter in Section 5.2. To fully investigate the behavior of the patched plate under thermo-mechanical loading, we consider various normalized lengths of the patch, $L_{\mathrm{p}}$, as well as various ratios of the coefficients of the thermal expansion, $\alpha_{0}=\alpha_{\mathrm{p}} / \alpha$, where $\alpha_{\mathrm{p}}$ and $\alpha$ are defined in Appendix B.

\subsection{Edges free to translate in-plane}

In this section we consider the case where the edges are free as to allow in-plane edge deflections. Detailed results for representative patched plates, which elucidate the characteristic behavior of the class of structures of interest, are presented and discussed in Section 5.1.1. These results are extended to a broad range of structures in Section 5.1.2 by examining the behavior of the characteristic parameters $N_{\mathrm{r}}$ and $\Theta_{\mathrm{cr}}$ for a wide range of structural properties.

\subsubsection{Loading scenarios}

For this case, we will be concerned with three types of loading scenarios: (i) a plate subjected to a constant temperature field and a controlled inplane edge force, (ii) a plate subjected to a controlled in-plane edge force and a controlled uniform (negative) temperature such that the loading parameter maintains a constant value, and (iii) a plate subjected to a constant in-plane edge force and a controlled uniform (negative) temperature field. For brevity, we limit our discussion to the representative cases where the ratio of thermal expansion coefficients is $\alpha_{0}=\frac{1}{2}, 1$ and 2 . We likewise restrict the current discussion to the representative case of patches of length $L_{\mathrm{p}}=0.8$ and thicknesses $h_{\mathrm{p}}=h=0.05$. Similar results are found when the structural and thermal parameters are varied, but are omitted for brevity.

5.1.1.1. Edge force controlled loading in a fixed temperature field. We consider the case where the plate is loaded with an in-plane edge force under a constant (negative) temperature field. The normalized membrane force, $N_{0}$, is displayed as a function of the in-plane edge-deflection, $u \equiv u_{2}(1)$, for hinged and clamped edge conditions in Figs. 2 and 3 , respectively, for a range of temperatures. (We recall that "temperature" in this context corresponds to the normalized temperature change, as defined in Section 2.) In Figs. $2 \mathrm{a}$ and $3 \mathrm{a}$ the load paths are displayed for $\alpha_{0}=\frac{1}{2}$, in Figs. $2 \mathrm{~b}$ and $3 \mathrm{~b}$ for $\alpha_{0}=1$, and in Figs. 2c and 3c for $\alpha_{0}=2$. For each case it may be seen, upon following an isotherm, that for vanishing membrane force, there will be an initial in-plane edge deflection which is 

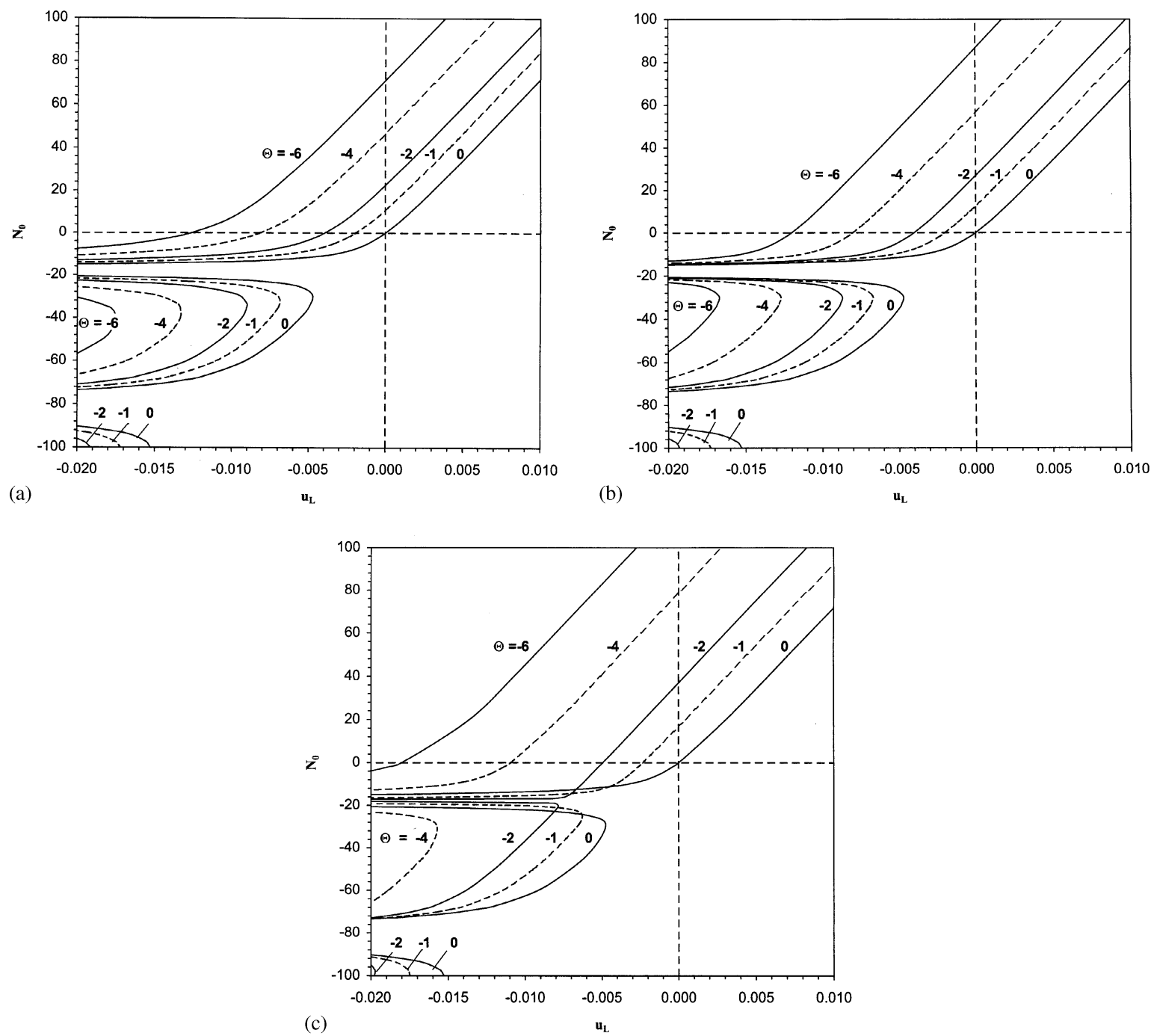

Fig. 2. Normalized membrane force, $N_{0}$, versus normalized in-plane edge displacement, $u$, for various normalized temperatures, $\Theta$; (a) $\alpha_{0}=\frac{1}{2}$, (b) $\alpha_{0}=1$, (c) $\alpha_{0}=2$. (Hinged supports, $L_{\mathrm{p}}=0.8$ ).

negative (compressive, $u<0$ ). This corresponds to the deformation due to thermal effects alone. Consider first the case when a tensile in-plane force is applied $\left(N_{0}>0\right)$. It may be seen that as the membrane force increases, the in-plane edge deflection increases, eventually achieving positive deflection. For large enough membrane force it may be seen that the in-plane deformation varies (in effect) linearly with increasing force, leaving only a small region where the relationship between the membrane force and the in-plane edge deflection is noticeably "non-linear". In the case of compressive membrane force this is not the case, as we will now discuss.

If the in-plane edge force is instead compressive $\left(N_{0}<0\right)$ it may be seen from Figs. 2 and 3, upon following an isotherm, that $u$ decreases with increasing magnitude of the compressive membrane 

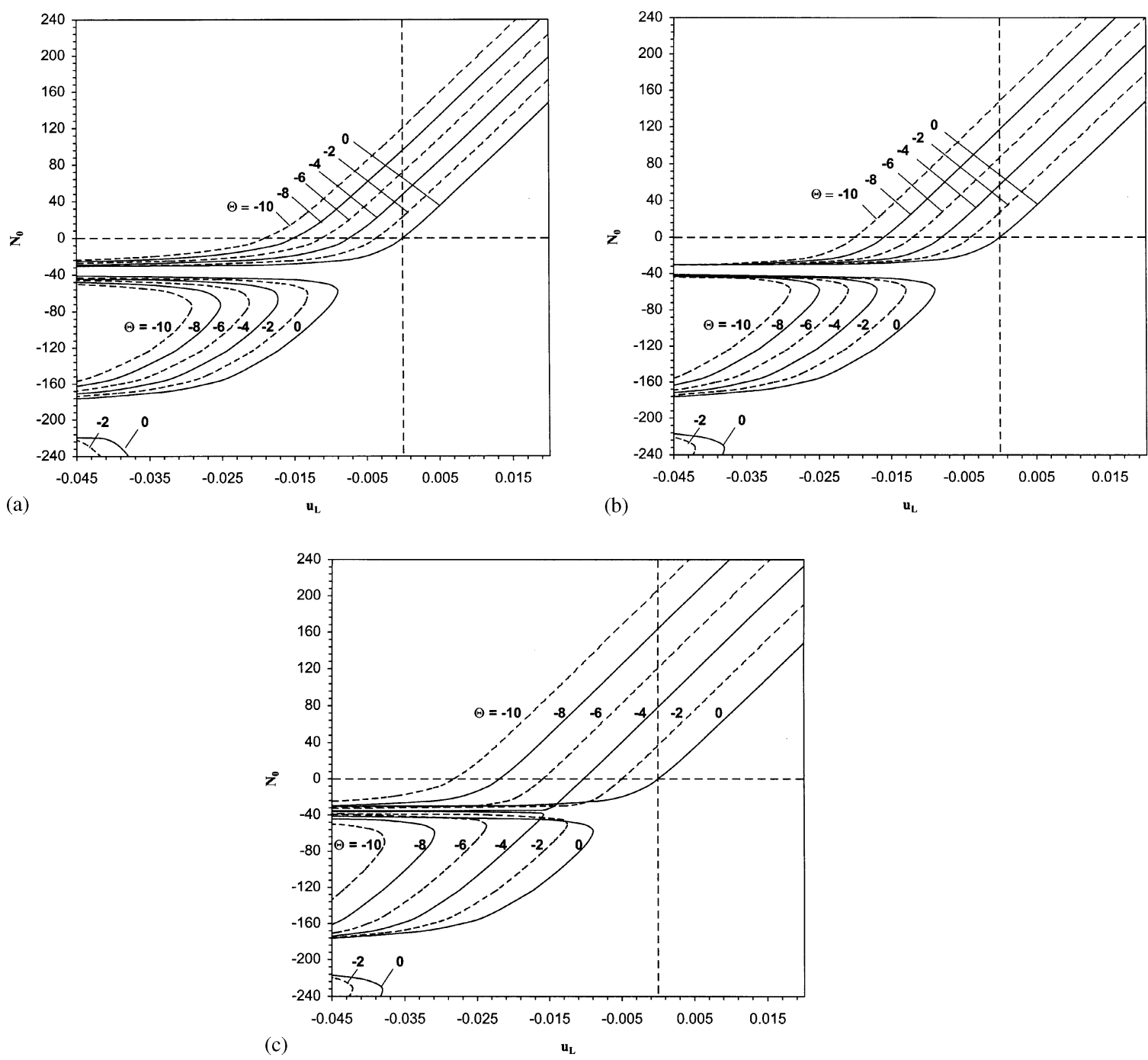

Fig. 3. Normalized membrane force, $N_{0}$, versus normalized in-plane edge displacement, $u$, for various normalized temperatures, $\Theta$; (a) $\alpha_{0}=\frac{1}{2}$, (b) $\alpha_{0}=1$, (c) $\alpha_{0}=2$. (Clamped supports, $L_{\mathrm{p}}=0.8$ ).

force. When a certain level of the compressive membrane force is approached, the edge deflection is seen to change in a relatively rapid fashion with increasing compressive force, with the force evidently approaching a limiting value. We refer to this type of behavior as "asymptotic buckling". The "limiting force" may be seen to be independent of $\Theta$ and $\alpha_{0}$, and corresponds to a buckling load of the system. It is equivalent to the critical membrane force, $N_{\mathrm{r}}$, as defined in Section 3 of this study and is found to have the numerical values of $N_{\mathrm{r}}=-17.8$ for hinged support conditions and $N_{\mathrm{r}}=-36.3$ for clamped support conditions. ${ }^{5}$ Thus, the critical membrane force as defined by Eq. (19) is seen to

\footnotetext{
${ }^{5}$ It may be seen in Figs. 2 and 3 that a second "limiting force" may be found at $N_{\mathrm{r}}=-81.3$ and -194.6 , respectively, which corresponds to the second critical membrane force. However, it will be seen next that for edge force controlled loading, compressive membrane forces with magnitude exceeding the lowest critical value may not be reached in the case of kinematically free boundaries, unless the system is artificially constrained until the first $N_{\mathrm{r}}$ is surpassed.
} 


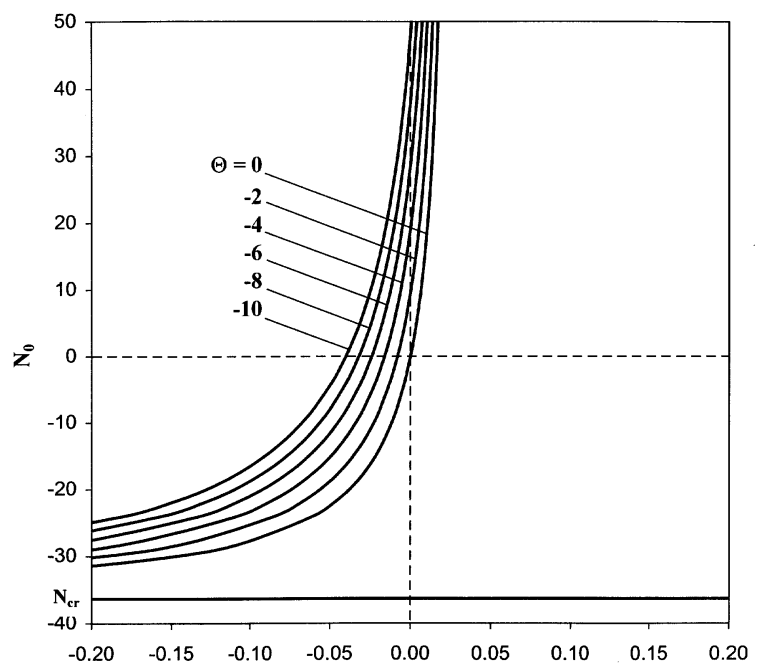

(a)

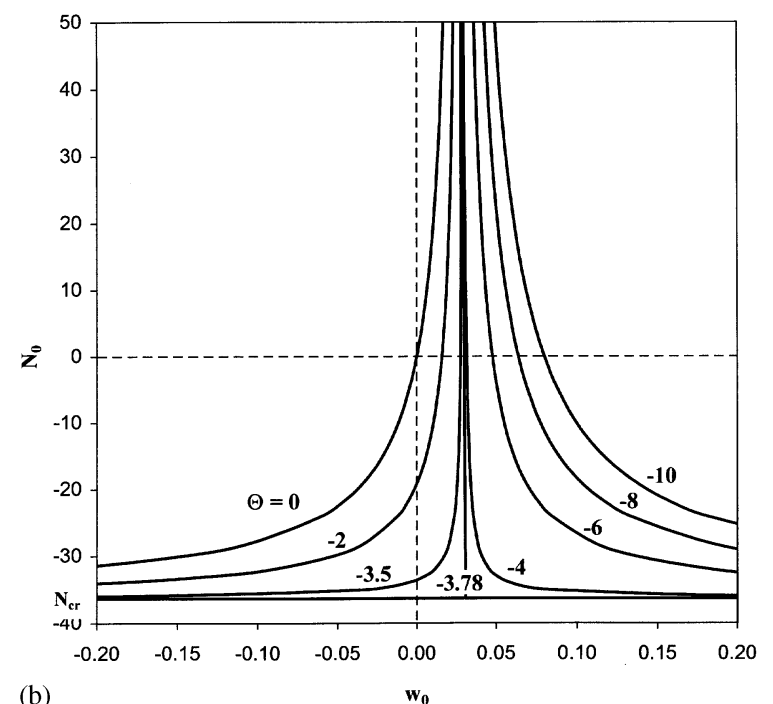

(b)

Fig. 4. Normalized membrane force, $N_{0}$, versus normalized transverse centerspan displacement, $w_{0}$, for various values of the normalized temperatures, $\Theta$; (a) $\alpha_{0}=\frac{1}{2}$, (b) $\alpha_{0}=2$. (Clamped-free supports, $L_{\mathbf{p}}=0.8$ ).

correspond to a buckling load of a structure under compressive force controlled edge loading as suggested in Section 3.

Consider next the corresponding transverse centerspan deflections. As indicated for the associated in-plane deflections, the general qualitative behavior is independent of the manner in which the edges are supported with regard to rotations. Hence, we limit our discussion to the case of clamped edges, for brevity. In Figs. $4 a$ and $b$ the membrane force, $N_{0}$, is displayed as a function of the center-span deflection $w_{0} \equiv w_{1}^{*}(0)$ for a range of temperatures, for the ratio of thermal expansion coefficients $\alpha_{0}=\frac{1}{2}$ and 2 , respectively. We recall that the critical membrane force for this case is $N_{\mathrm{r}}=-36.3$. Consider the latter case first $\left(\alpha_{0}=2\right.$, Fig. 4b). For vanishing membrane force $\left(N_{0}=0\right)$, it may be seen for each $\Theta$ that the centerspan has an initial positive deflection (downwards), corresponding to the temperature effects alone. When the edges of the plate are subsequently loaded with a compressive in-plane force it may be seen that as the magnitude of the compressive membrane force increases the centerspan deflection changes first moderately. As the value of the edge load approaches $N_{\mathrm{r}}$ the deflection becomes large and the structure in effect buckles ("asymptotic buckling"), as may be anticipated from the discussion concerning the in-plane deflection. However, the sense of the centerspan deflection, as $N_{\mathrm{r}}$ is approached, is seen to depend on the magnitude of the temperature. For the structure under consideration, and with $\alpha_{0}=2$, the critical temperature is given by $\Theta_{r}=-3.78$. It may be seen that if the temperature field is larger than the critical temperature $(0>\Theta>-3.78)$ the centerspan will, for large enough $N_{0}$, deflect upwards (negative values of $w_{0}$ ), and for $\Theta<-3.78$ the centerspan will deflect downwards. When $\Theta=\Theta_{\mathrm{r}}$, the centerspan deflection will change very little until the critical membrane force is reached. At this point the deflection becomes undetermined, within the context of the present mathematical model, and bifurcation buckling occurs. Thus, it may be concluded that the critical temperature divides the structural behavior between upward and downward deflection changes. ${ }^{6}$ It may further be noted that the magnitude

\footnotetext{
${ }^{6}$ Similar results are found for a variety of combinations of relative patch lengths and ratios of coefficients of thermal expansion. Likewise, similar behavior is observed for structures with hinged edges. Such results are omitted for brevity.
} 
of the compressive membrane force may not exceed the magnitude of the critical membrane force in the loading scenario described.

If the structure is instead loaded with a tensile edge force $\left(N_{0}>0\right)$, it may be seen from Fig. $4 \mathrm{~b}$ that the structure will not buckle. For any combination of temperature and tensile membrane force the centerspan deflection will be positive. It may be noted that as the tensile force increases the centerspan deformation is seen to approach the deflection corresponding to that of the critical temperature asymptotically.

It was shown in Section 3 that for structures where $\alpha_{0} \leqslant 1$ (i.e., $\left.m^{*} \leqslant 0\right)^{7}$ a (negative) critical temperature does not exist. For the limiting case where $\alpha_{0}=1\left(m^{*}=0\right)$, it may be seen from Eq. (13) that the load parameter depends on the membrane force alone. Thus, for this case, the transverse deflection of the plate is independent of the temperature. It may be easily verified from Eqs. (13), (14a), (14b), (15), (16a), (16b), (17), (18a) (18b), (18c), (18d) and (18e) that the plate deflects upwards if a compressive in-plane edge load is applied and downwards if a tensile in-plane edge load is applied, where the latter was seen for vanishing temperature in Ref. [5]. Upon consideration of Fig. 4a, which displays the membrane force as a function of the transverse centerspan deflection for a range of temperatures for $\alpha_{0}=\frac{1}{2}$, it may be seen that the structure deflects upwards for compressive edge loads, for all temperatures considered. If this structure is instead loaded with a tensile edge force, it may be seen that for lower tensile forces the structure will exhibit upward deflections. However, as the tensile force is increased, the centerspan deflection bends downward. There will be one point for each given isotherm where the centerspan deflection vanishes. This corresponds to a loading combination yielding vanishing loading parameter, and thus the transverse deflection vanishes uniformly for the structure at this point.

The different directions of the transverse deflection for the loading scenarios considered may be

\footnotetext{
${ }^{7}$ It follows from Eqs. (B.3), (B.5h), (B.5i) and (B.5j) that if $\alpha_{0}=1$ then $m^{*}=0$, if $\alpha_{0}<1$ then $m^{*}<0$, and if $\alpha_{0}>1$ then $m^{*}>0$.
}

explained in the following manner. Consider a patch that has a coefficient of thermal expansion larger than that of the base structure (i.e., $\alpha_{0}>1$ ). If the structure is subjected to a temperature field alone (vanishing membrane force), the mismatch in thermal expansion coefficients leads to downward deflections of the plate (i.e., $w$ positive). This is supported by Fig. $4 \mathrm{~b}\left(\alpha_{0}=2\right)$, when $N_{0}=0$. However, when the plate is loaded with only a compressive membrane force (vanishing temperature), the plate will tend to deflect upward ( $w$ negative). This again is supported by Fig. $4 \mathrm{~b}$, for the isotherm $\Theta=0, N_{0}<0$. When the plate is loaded with a combination of compressive membrane force and temperature, the tendency for the temperature to induce positive deflection "competes" with the tendency for the compressive membrane force to induce negative deflection. When $\Theta>\Theta_{r}$ the membrane force is seen to prevail, and hence it governs the direction of the transverse deflection. For $\Theta<\Theta_{r}$ the opposite is true. In the case of a vanishing load parameter $\left(\mathscr{M}_{\lambda}=0\right)$, the deflections associated with temperature and those associated with the membrane force cancel one another, producing vanishing transverse displacement. When the structure is instead loaded with a tensile force, the deflections associated with temperature and with membrane force, reinforce each other and hence induce deflections in the same direction. If we next consider the structures where the patch has a lower or equal coefficient of thermal expansion to that of the base structure, $\alpha_{0} \leqslant 1$, we have seen that no negative critical temperature exists. If these structures are loaded with a temperature load only, the structure will bend upwards. This may be seen in Fig. $4 \mathrm{a}$, for $N_{0}=0$. Following the same reasoning as above, it may be seen that when this structure is loaded with a compressive edge force, the transverse deformation will be reinforced to deform upwards. However, for the case of a tensile edge force, the tendency for a tensile membrane force to cause upward bending will overtake the temperature effects monotonically as the edge force is increased. In this case there will be no "critical behavior", since a tensile membrane force does not yield any critical membrane force and thus no critical temperature (as defined in Eq. (20)). 
5.1.1.2. Edge force controlled loading with fixed loading parameters. Let us consider the case where the temperature, $\Theta$, and membrane force, $N_{0}$, are controlled in such a manner that the loading parameter, $\mathscr{M}_{\lambda}$, will remain constant. In particular, if $N_{0}$ is changed monotonically, the value of $\Theta$, or more generally the value of $m^{*} \Theta$, required to maintain $\mathscr{M}_{\lambda}$ at a constant value may be determined by Eq. (13). In Fig. 5, the membrane force, $N_{0}$, is displayed as a function of the centerspan deflection, $w_{0}$, for various values of the load parameter, $\mathscr{M}_{\lambda}$. The path corresponding to the case of $m^{*} \Theta=0$ is also displayed. Depending on the value of $m^{*}$, Fig. 5 may be interpreted as described in the following.

If the composite structure under consideration corresponds to one where $m^{*}<0\left(\alpha_{0}<1\right)$ then all load paths, or portions of load paths, that are located above and to the left of $m^{*} \Theta=0$ correspond to negative temperatures. The load paths below and to the right of $m^{*} \Theta=0$ correspond to positive temperatures, and are thus not considered in this study. Obviously, in this case, the curve $m^{*} \Theta=0$ must correspond to $\Theta=0$. If instead the composite structure corresponds to one where

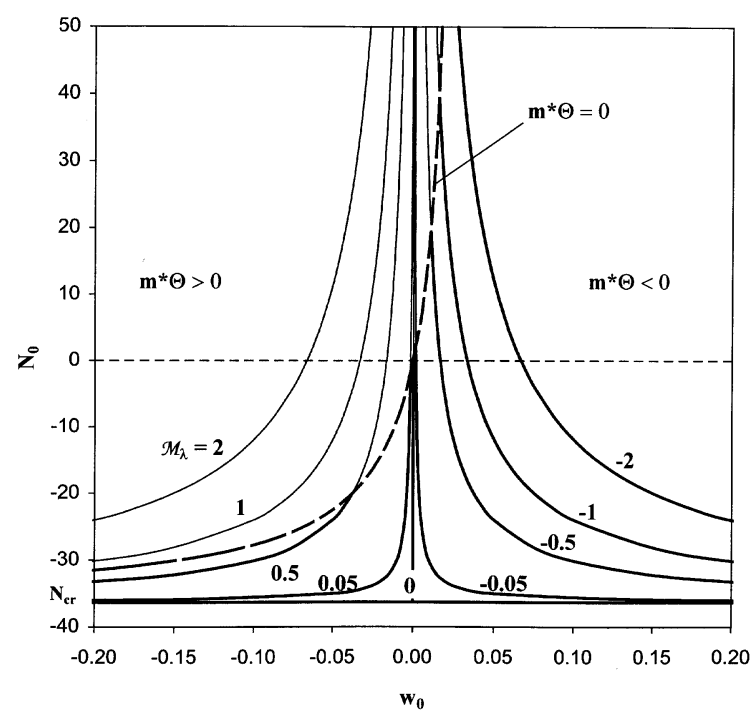

Fig. 5. Normalized membrane force, $N_{0}$, versus normalized transverse centerspan displacement, $w_{0}$, for various values of the loading parameter, $\mathscr{M}_{\lambda}$. (Clamped-free supports, $\left.L_{\mathrm{p}}=0.8\right)$. $m^{*}>0\left(\alpha_{0}>1\right)$ the opposite is true; paths corresponding to negative temperatures are situated below and to the right of the curve $m^{*} \Theta=0$ (i.e., $\Theta=0$ ). For the case when the patch has the same coefficient of thermal expansion as the base structure $\left(m^{*}=0\right.$ and equivalently $\left.\alpha_{0}=1\right)$ the curve $m^{*} \Theta=0$ corresponds to $m^{*}=0$. Thus, for this case, the transverse displacement as a function of increasing membrane force is given by the curve $m^{*} \Theta=0$ and it may be seen that a constant value of the loading parameter cannot yield permissible solutions for this case.

With the above described interpretations of Fig. 5 in mind, we may consider the general behavior as characterized by the centerspan deflections, $w_{0}$, for a constant loading parameter, $\mathscr{M}_{\lambda}$, and a monotonically changing membrane force, $N_{0}$. It was seen in Section 3 that a vanishing loading parameter corresponds to vanishing transverse deflection. This is supported in Fig. 5 by a vanishing centerspan deflection for $\mathscr{M}_{\lambda}=0$ for all membrane forces considered. For this case, $N_{0}=N_{\mathrm{r}}$, corresponds to a bifurcation point. Thus, if the structure is loaded with a compressive normal force $\left(N_{0}<0\right)$, bifurcation buckling occurs once the critical membrane force is achieved. For non-vanishing loading parameters, it may be seen that $\mathscr{M}_{\lambda}<0$ corresponds to downward deflections of the structure, while $\mathscr{M}_{\lambda}>0$ corresponds to upward deflections. Furthermore, it may be noted that the centerspan deflection is symmetric with respect to the magnitude of the loading parameter. It may be seen that if the structure is loaded with a compressive edgeforce and $\mathscr{M}_{\lambda} \neq 0$, the deflections become large as the critical membrane force is approached so that the structure, in effect, buckles ("asymptotic buckling") and $N_{\mathrm{r}}$ is never exceeded. ${ }^{8}$

5.1.1.3. Temperature controlled loading. We next consider the case where the plate is subjected to a constant in-plane edge force and loaded with a uniformly decreasing temperature field. Figs. $6 \mathrm{a}-\mathrm{c}$

\footnotetext{
${ }^{8}$ Comparing the present results to the classical results for (unpatched) beam-plates, it may be seen that the magnitude of $\mathscr{M}_{\lambda}$ describes how far the structure is from being "perfect", with $\mathscr{M}_{\lambda}=0$ corresponding to a "perfect" structure.
} 

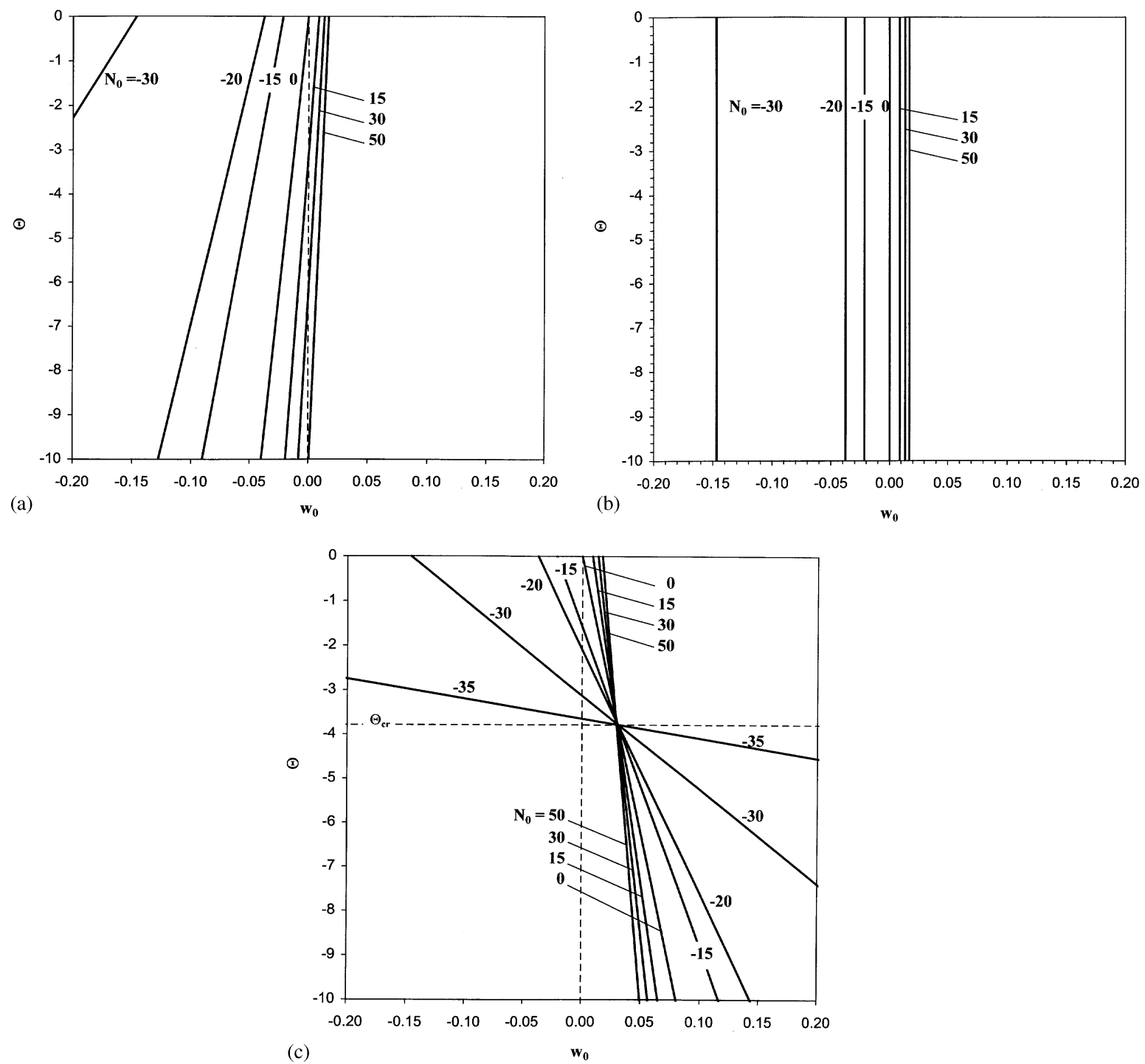

Fig. 6. Normalized temperature, $\Theta$, versus normalized transverse centerspan displacement, $w_{0}$, for various values of the normalized membrane force, $N_{0}$; (a) $\alpha_{0}=\frac{1}{2}$, (b) $\alpha_{0}=1$, (c) $\alpha_{0}=2$. (Clamped-free supports, $L_{\mathrm{p}}=0.8$ ).

display the temperature, $\Theta$, as a function of the centerspan deflection, $w_{0}$, for a range of values of the normalized membrane force, $N_{0}$, for the ratios of thermal expansion coefficients $\alpha_{0}=\frac{1}{2}, 1$ and 2, respectively. For all cases, it may be seen, that for a plate at its reference temperature $(\Theta=0)$, the centerspan deflection is negative (the plate deflects upwards) for compressive in-plane edge loads and positive for tensile edge loads.
Consider first the case of $\alpha_{0}=\frac{1}{2}$, displayed in Fig. 6a. It may be seen that for compressive loading the magnitude of the centerspan deflection increases as the temperature decreases (i.e. the structure deforms further upwards). If the plate instead is loaded with a constant tensile edge-force it may be seen that as the plate is continuously cooled, the centerspan deflection changes from being bent downward to being bent upwards. For the case of 
$\alpha_{0}=1$, it may be seen in Fig. $6 \mathrm{~b}$ that the centerspan deflection is independent of the temperature, as expected. In Fig. 6c, the case of $\alpha_{0}=2$ is shown. For the case of compressive edge forces, it may be seen that as the temperature decreases, the magnitude of the upward centerspan deflection decreases and eventually the structure will be deflected downwards. When the structure is instead loaded with a tensile edge load, the centerspan deflects upwards and the magnitude increases as the structure is cooled. ${ }^{9}$ In Figs. $6 \mathrm{a}$ and $\mathrm{c}$ it may be seen that the closer the constant membrane force is to the critical membrane force, $N_{\mathrm{r}}=-36.3$, the more sensitive the deformation is to changes in the temperature.

5.1.1.4. Synopsis. The numerical simulations presented above have demonstrated that the critical temperature and the critical membrane force are of great importance to the structural behavior of an edge-loaded patched plate with translationally free edges subjected to decreasing temperature. The critical temperature is related to bifurcation of the equilibrium path for a structure in a constant temperature field or with a constant loading parameter. The critical membrane force is seen to correspond to the structure's "buckling load". The membrane force is seen to generally approach, but never exceed the critical membrane force ("asymptotic buckling"). The qualitative behavior seen for the particular structural parameters considered in this section are characteristic of all structures of this class, within the context of the formulation presented in Section 2. For other patch lengths, structural stiffnesses, and thicknesses, variation in critical behavior may be characterized by examination of corresponding variations of the critical temperature and critical membrane force. The behavior of these parameters for various structures is discussed in the next subsection.

\footnotetext{
${ }^{9}$ In Fig. $6 \mathrm{c}\left(\alpha_{0}=2\right)$, all paths appear to cross each other at the critical temperature within the resolution of the figure. This is not actually the case, as would be seen upon magnification in the vicinity of the apparent crossing. Though they appear close, the deflections at $\Theta=\Theta_{r}$ are not independent of the membrane force.
}

\subsubsection{Critical parameters}

In this subsection the characteristics of the critical membrane force, $N_{\mathrm{r}}$, and the critical temperature, $\Theta_{r}$, are discussed. These two parameters were seen above to be of critical importance for the response of the composite system considered.

An investigation of the critical membrane force was conducted in Ref. [1]. It was therein seen that the membrane forces associated with antisymmetric deformation yield significantly higher values of the critical membrane force than do their symmetric counterparts. Thus, if the structure is loaded from the trivial state, the antisymmetric load cannot be achieved (unless the system is artificially constrained until the lower $N_{\mathrm{r}}$ is surpassed). The quantitative results for the critical membrane force are not repeated herein for brevity. However, it was seen that the magnitude of the (lowest) critical membrane force increases with increasing patch length and that it is always larger for the case of clamped supports than for of the case of hinged supports, as might be expected.

For the present case, the critical temperature, $\Theta_{r}$, is presented as functions of the structural properties of the patched plate. These properties include the relative length of the patch, the normalized thickness of the base plate, and the modulus ratios of the composite structure. In each case, the thickness ratio is maintained at unity $\left(h_{\mathrm{p}}=h\right)$. In order to vary the relative stiffness of the patch, several orders of magnitude of the modulus ratio, $E_{0}$, are considered. In this way, the behavior of a broad range of structures is characterized.

In Figs. 7 and 8 the critical temperature, $\Theta_{r}$, corresponding to the lowest critical membrane force for symmetric deformation is displayed as a function of $\alpha_{0}$, for hinged and clamped edge conditions, respectively. In Figs. 7a and 8a results are shown for various values of the modulus ratio, $E_{0}$, for $h=0.05$. We recall that when $\alpha_{0}<1$, no (negative) critical temperature exists. It may be seen that for a given $\alpha_{0}$ the critical temperature, $\Theta_{\mathrm{r}}$, decreases (higher negative values) with increasing stiffness. For values of $\alpha_{0}$ close to 1 the values of the critical temperature appear to be asymptotic to the line $\alpha_{0}=1$. As $\alpha_{0}$ increases, it may be seen that $\Theta_{\mathrm{r}}$ approaches zero. Figs. $7 \mathrm{~b}$ and $8 \mathrm{~b}$ display $\Theta_{\mathrm{r}}$ for a range of patch lengths, and it may be seen 

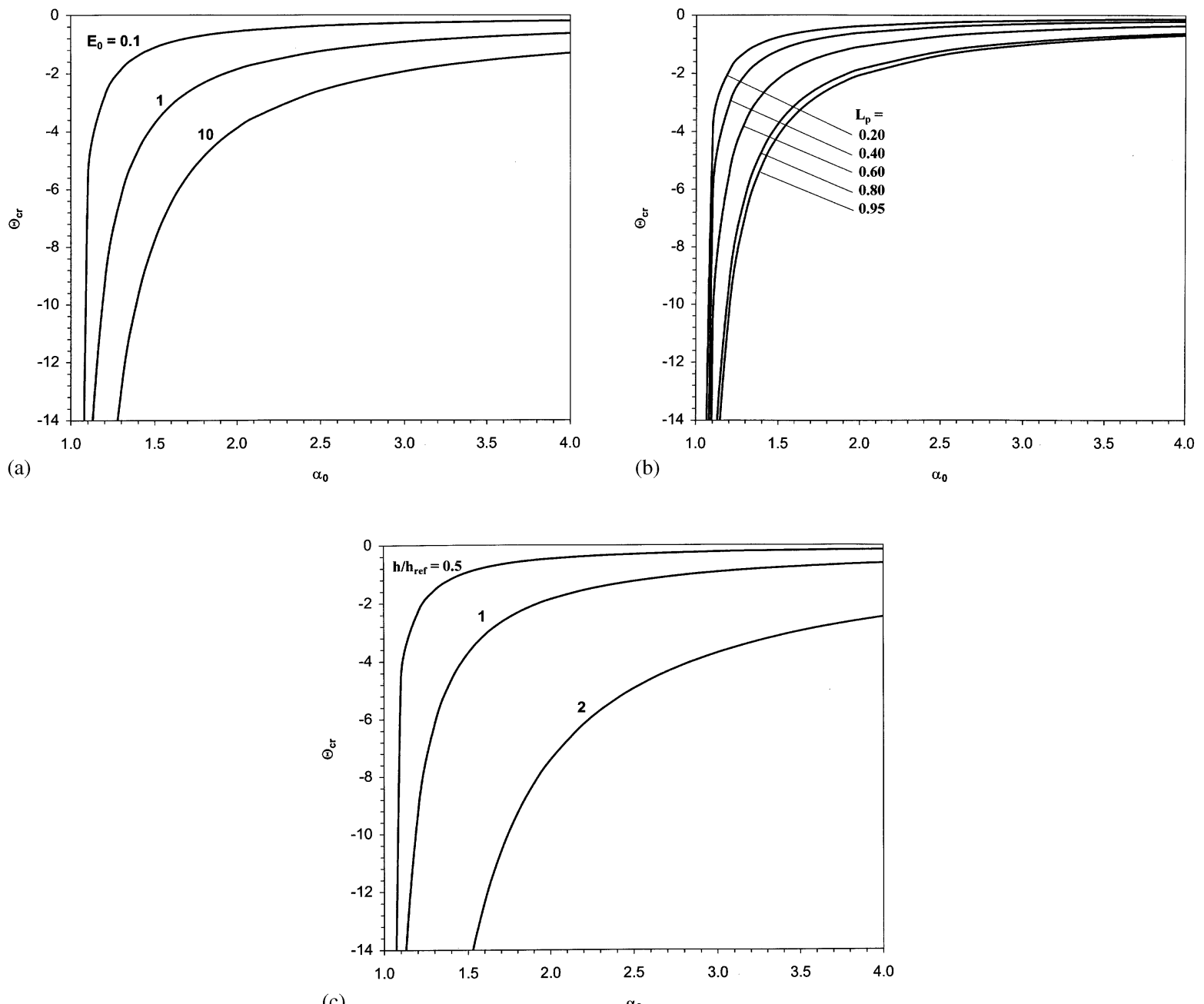

(c)

Fig. 7. The critical temperture, $\Theta_{\mathrm{r}}$, corresponding to the first critical membrane force for symmetric deformation versus the ratio of coefficients of thermal expansion, $\alpha_{0}$, for hinged supports; (a) various relative stiffnesses $\left(L_{\mathrm{p}}=0.8, h=0.05\right.$ ), (b) various patch lengths $\left(E_{0}=1, h=0.05\right),(\mathrm{c})$ various thicknesses $h_{\text {ref }}=0.05\left(L_{\mathrm{p}}=0.8, E_{0}=1\right)$.

that $\Theta_{\mathrm{r}}$ decreases with increasing patch lengths. In Figs. $7 \mathrm{c}$ and $8 \mathrm{c}, \Theta_{\mathrm{r}}$ is shown for various values of $h$, where $h_{\text {ref }}=0.05$. It may be seen from these figures that as $h$ increases, the critical temperature decreases.

\subsection{Edges fixed against in-plane translation}

We next consider the case where the edges of the base plate are fixed so as to prohibit in-plane trans- lation, and consider temperature controlled loading. Both hinged and clamped (rotational) support conditions are considered. For brevity, we (initially) limit our discussion to the representative cases where the ratio of coefficients of thermal expansion is $\alpha_{0}=\frac{1}{2}, 1$ and 2. We likewise restrict the current discussion to the representative case of patches of thicknesses $h_{\mathrm{p}}=h=0.05$, and for modulus ratio $E_{0}=1$. After the qualitative response of the composite structure has been established, the discussion 

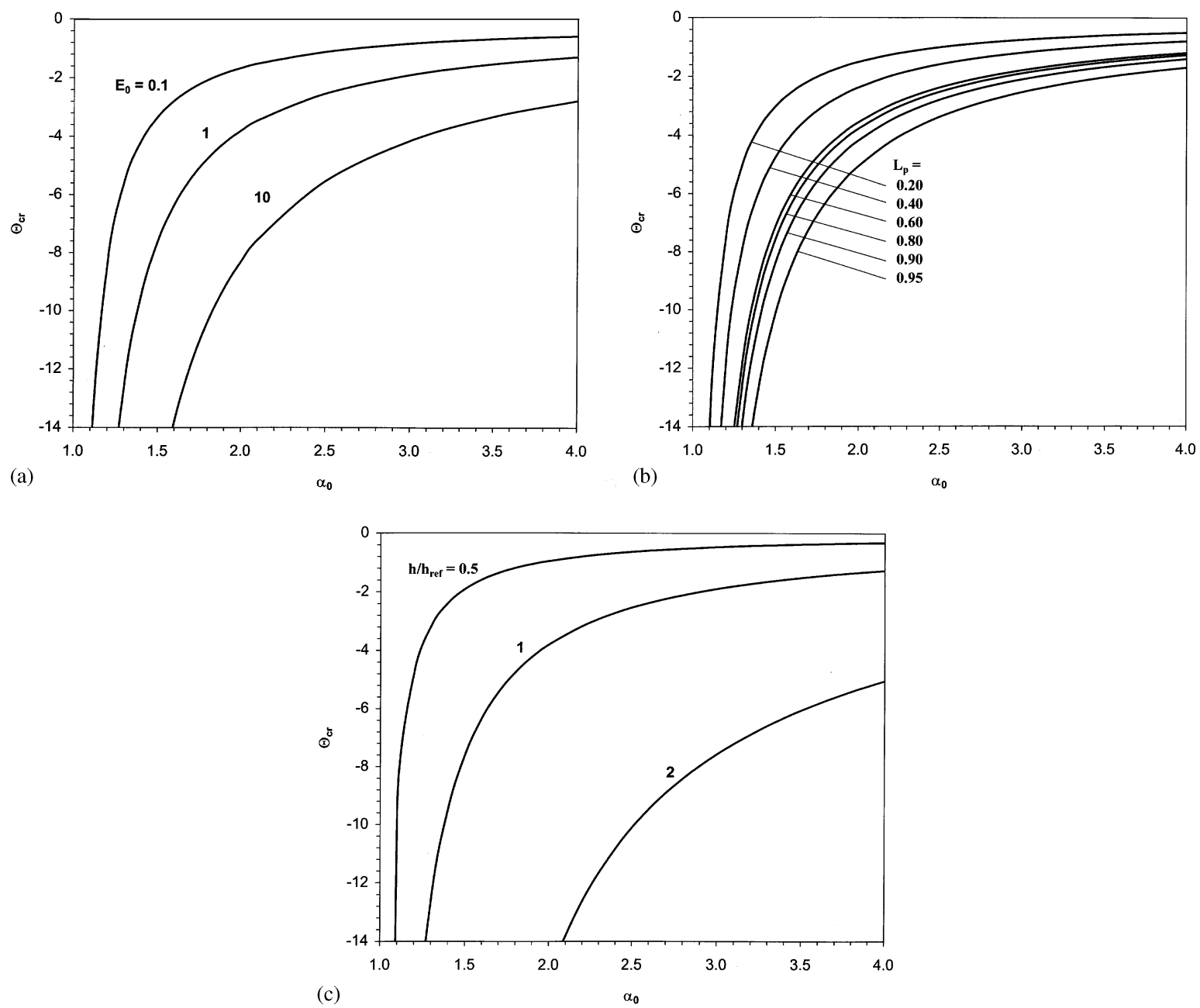

Fig. 8. The critical temperture, $\Theta_{\mathrm{r}}$, corresponding to the first critical membrane force for symmetric deformation versus the ratio of coefficients of thermal expansion, $\alpha_{0}$, for clamped supports; (a) various relative stiffnesses $\left(L_{\mathrm{p}}=0.8, h=0.05\right)$, (b) various patch lengths $\left(E_{0}=1, h=0.05\right),(\mathrm{c})$ various thicknesses $h_{\text {ref }}=0.05\left(L_{\mathrm{p}}=0.8, E_{0}=1\right)$.

is extended to structures with a wide range of structural properties. ${ }^{10}$

We recall from prior discussion that only discrete combinations of membrane forces and temperatures correspond to equilibrium configurations of the composite structure when in-plane translation

\footnotetext{
${ }^{10}$ It was shown in Section 3 that antisymmetric solutions may only exist when the edges are free to allow in-plane deflections, thus only symmetric solutions need to be considered.
}

is prohibited at the edges of the base plate. Specifically, the membrane force, $N_{0}$, is solved numerically, as roots to the integrability condition, Eq. (11), with $u_{2}(1)=0$ for each given temperature, $\Theta$. We may recall that the in-plane displacements of structures with patch length $L_{\mathrm{p}}=0.8$ are given in Figs. 2 and 3, for the case of hinged and clamped supports, respectively. For a given temperature the corresponding membrane forces are found where the appropriate isotherm intercepts $u_{\mathrm{L}}=0$. It may be seen from the figures that there will only be one 


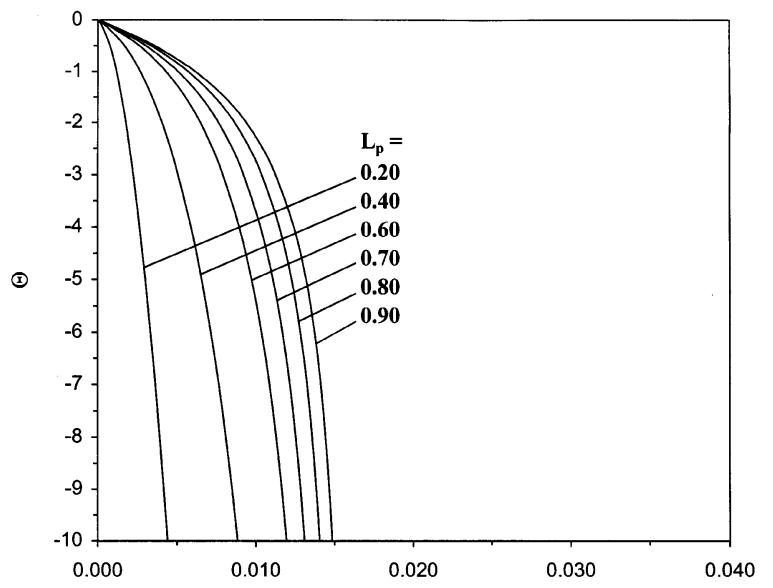

(a)

$\mathbf{w}_{0}$

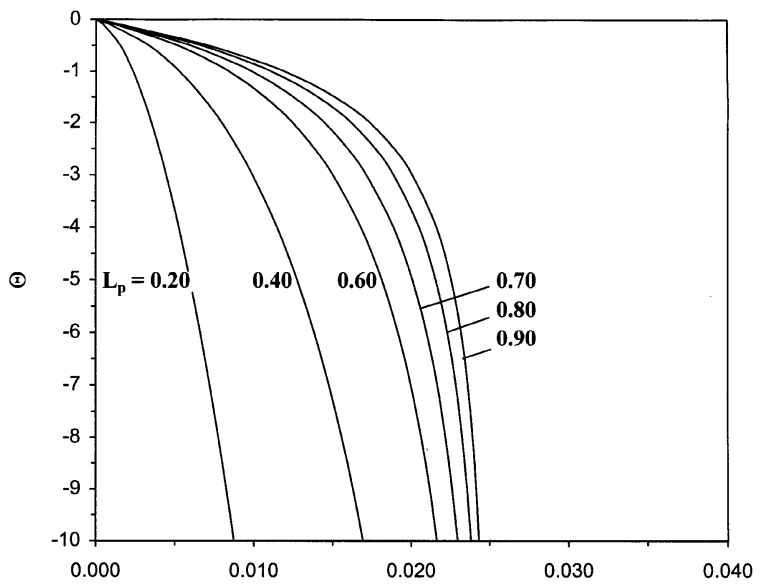

(b)

$\mathbf{w}_{0}$

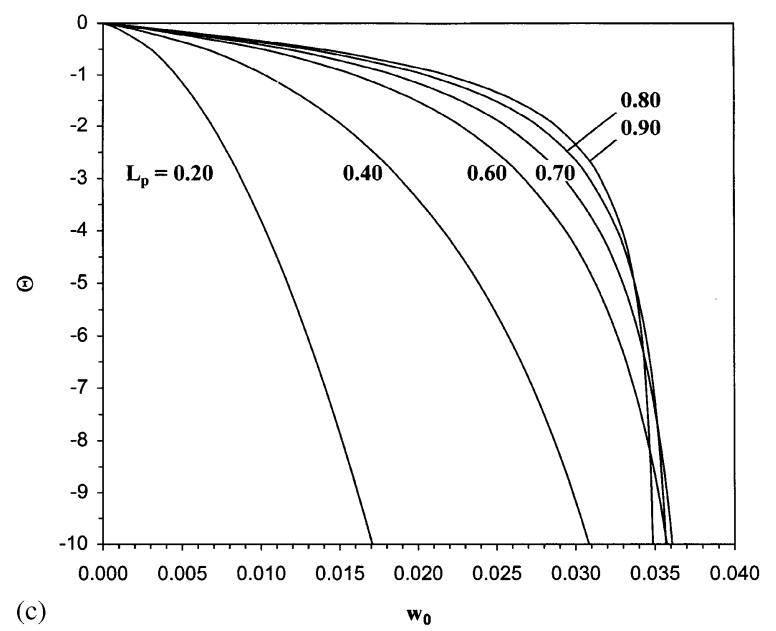

Fig. 9. Normalized temperature, $\Theta$, versus normalized transverse centerspan displacement, $w_{0}$, for various patch lengths, $L_{\mathrm{p}} ;\left(\right.$ a) $\alpha_{0}=\frac{1}{2}$, (b) $\alpha_{0}=1$, (c) $\alpha_{0}=2$. (Hinged-fixed supports).

possible membrane force for a given temperature within the range of temperatures considered. Since this implies that only one equilibrium configuration is possible for each given temperature (within the given range), stability is not an issue for the present case.

With the above established, the response of the composite system during cooling is characterized as follows. The temperature, $\Theta$, is displayed as a function of the centerspan deflection, $w_{0}$, for a range of patch lengths in Figs. 9 and 10 for hinged and clamped edge conditions, respectively. Figs. 9a and $10 \mathrm{a}$ correspond to the ratio of thermal coefficients $\alpha_{0}=\frac{1}{2}$, Figs. $9 \mathrm{~b}$ and $10 \mathrm{~b}$ to $\alpha_{0}=1$ and Figs. $9 \mathrm{c}$ and $10 \mathrm{c}$ to $\alpha_{0}=2$. It may be seen from the figures that for negative temperatures the centerspan deflection is positive (downward) for all cases considered. In the spirit of earlier discussions, it is found that, for this case, the mechanical component of the loading parameter, $\mathscr{M}_{\lambda}$, either dominates or reinforces the thermal component of $\mathscr{M}_{\lambda}$, as per Eq. (13) and as demonstrated in Fig. 11 for clamped supports. (Results for hinged support conditions yield similar results but are omitted for 

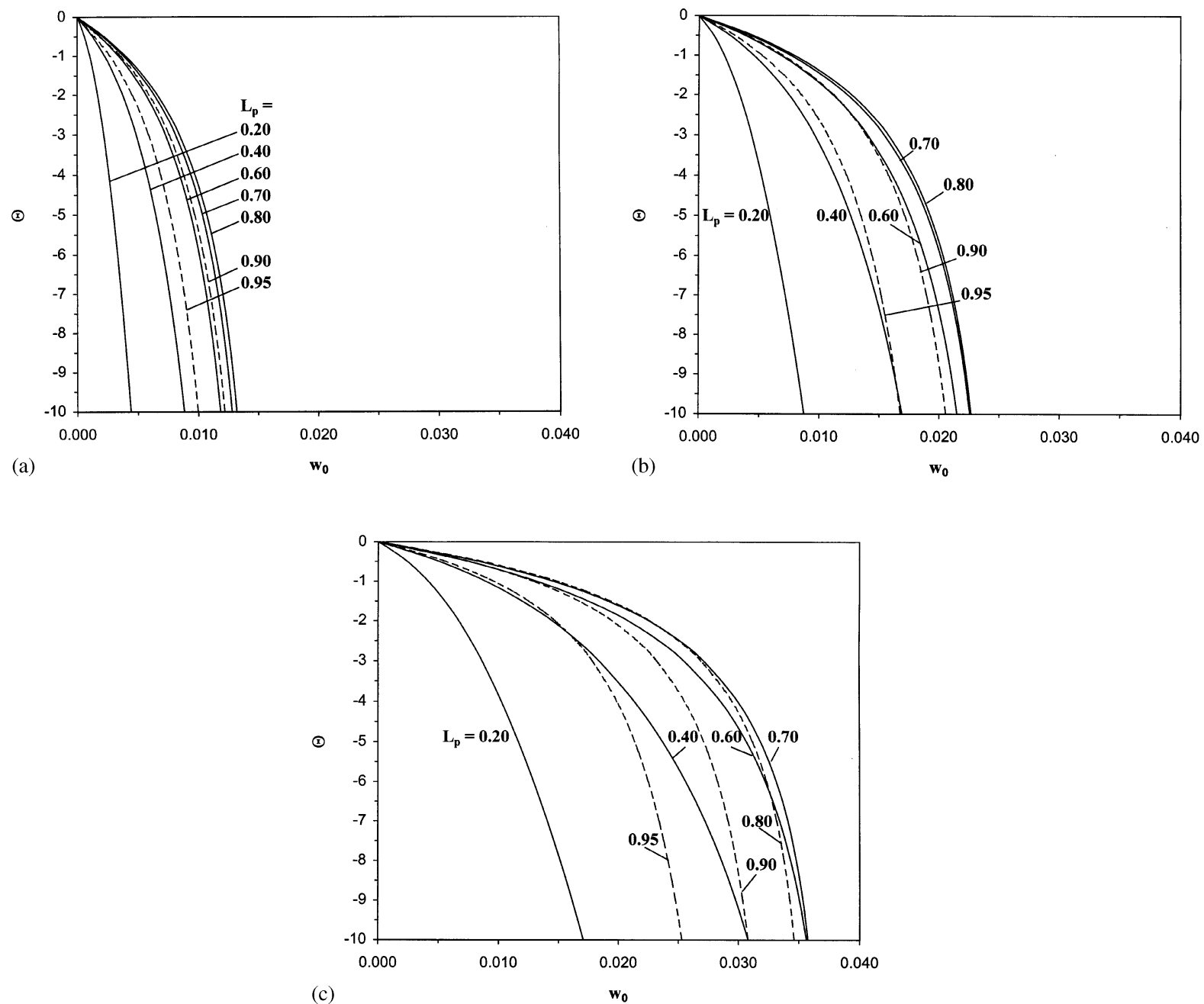

Fig. 10. Normalized temperature, $\Theta$, versus normalized transverse centerspan displacement, $w_{0}$, for various patch lengths, $L_{\mathrm{p}} ;$ (a) $\alpha_{0}=\frac{1}{2}$, (b) $\alpha_{0}=1$, (c) $\alpha_{0}=2$. (Clamped-fixed supports).

brevity.) Furthermore, it may also be seen from Figs. 9 and 10 that, in general, the deflection increases with increasing $\alpha_{0}$ and increasing $L_{\mathrm{p}}$. This general trend makes sense, of course, since it is the disparity in thermal expansion coefficients, along with the sizes of the patch and base structures, that drives the deformation. (The more material that wants to contract at different rates, the more flexure that is induced.) The trend may be observed to reverse itself, however, for relatively long patches $\left(L_{\mathrm{p}} \geqslant 0.8\right)$ for base structures with clamped sup- ports (Figs. 10a-c). This may be attributed to the stiffening of the system introduced as the patch edge approaches the support (i.e., as $L_{\mathfrak{p}} \rightarrow 1$ ). To emphasize this point, the global stiffness of the system $K=\left|M_{\lambda}\right| /|\psi|$, where $\psi \equiv w^{\prime}\left(L_{\mathrm{p}}\right)$ is the corresponding generalized displacement associated with the loading parameter, is displayed as a function of the temperature, $\Theta$, for various patch lengths in Figs. 12a-c. Comparison of Figs. 12a-c with Figs. $10 \mathrm{a}-\mathrm{c}$ shows a direct correlation of the global stiffness with the aforementioned tendencies. This 


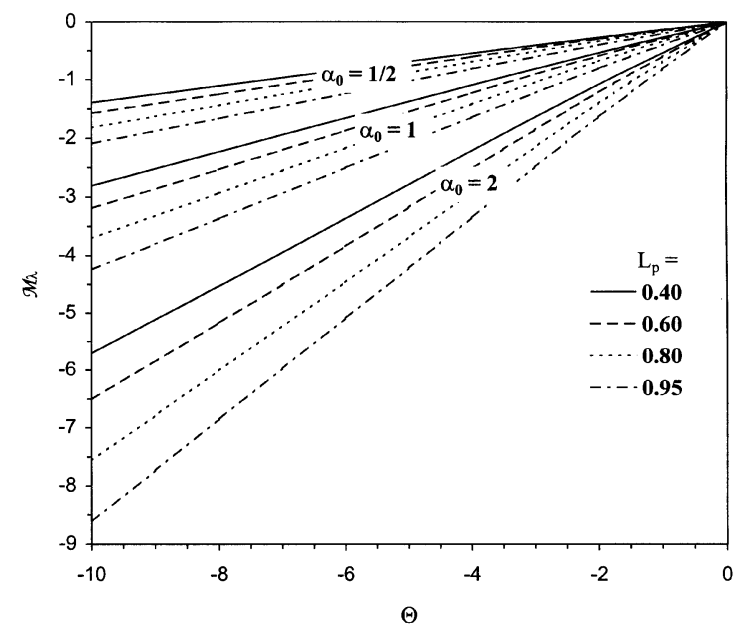

Fig. 11. Loading parameter, $\mathscr{M}_{\lambda}$, versus normalized temperature, $\Theta$, for various patch lengths, $L_{\mathrm{p}}$, and various ratios of coeffficients of thermal expansion, $\alpha_{0}$. (Clamped-fixed supports).

boundary induced stiffening effect is also seen to occur for structures with hinged supports (Fig. 9c) as $L_{\mathrm{p}} \rightarrow 1$, and may be attributed to (the resistance of) the relatively large deflection associated with $\alpha_{0}=2$ at sufficiently large $|\Theta|$.

With the qualitative behavior established, we now extend the results to a range of patched structures with edges prohibited from in-plane deformations. In particular we will characterize the structural response by presenting the centerspan deflection for various modulus ratios, $E_{0}$. (Varying $E_{0}$ by several orders of magnitude, while maintaining the thickness ratio at unity $\left(h_{\mathrm{p}}=h=0.05\right)$, corresponds to varying the relative stiffness of the structure. In this way we capture the behavior of a broad range of structures.) The centerspan deflection, $w_{0}$, is displayed as a function of $\alpha_{0}$ in Figs. 13a-c for patch lengths $L_{\mathrm{p}}=0.6,0.8$ and 0.9 , respectively, for $\Theta=-10$. Results for both hinged and clamped support conditions are displayed in each figure. It may be seen that in each case the centerspan deflection increases as the relative stiffness increases. An increase in relative stiffness increases both the thermal and mechanical components of the loading parameter and hence induces a larger centerspan deflection. Shorter patches show similar results but the results are omitted for brevity. It may be noted from Figs. 13a-c that the type of rotational support condition has a minor effect on the centerspan deflection. For shorter patches $\left(L_{\mathrm{p}}<0.6\right)$ it is found that the difference in the response for the two types of rotational supports, in effect, vanishes. Paralleling the discussion above regarding the centerspan deflection for longer patches, it follows that the stiffness from the supports tends to be less significant for shorter patches. Thus, it may be anticipated that the centerspan deflection is less sensitive to the rotational support conditions for the case of shorter patches.

\section{Concluding remarks}

The behavior of patched plates during cooling has been investigated. The response of the composite system to a uniform temperature field in combination with a membrane load was described through a self-consistent formulation, yielding a non-linear mathematical model. Numerical simulations based on analytical solutions were performed, thus results are exact within the context of the formulation.

Several non-dimensional parameters were identified. These were a loading parameter, consisting of a linear combination of the normalized membrane force and the normalized temperature, and two characteristic parameters, a critical temperature and a critical membrane force. All transverse deflections were seen to be proportional to the loading parameter, thus vanishing loading parameter was seen to correspond to flat configurations of the structure.

Results of numerical simulations were presented for representative patched plates to elucidate the characteristic behavior of the class of structures considered. It was seen that the type of supports regarding in-plane edged deflections has a significant influence on the response of the structure, while the type of rotational edge supports has less influence. For the case of the edges being free so as to allow in-plane translation, a representative patched plate was investigated. The results were extended to a broad range of structures by examining the behavior of the characteristic parameters for a variety of geometric and material 


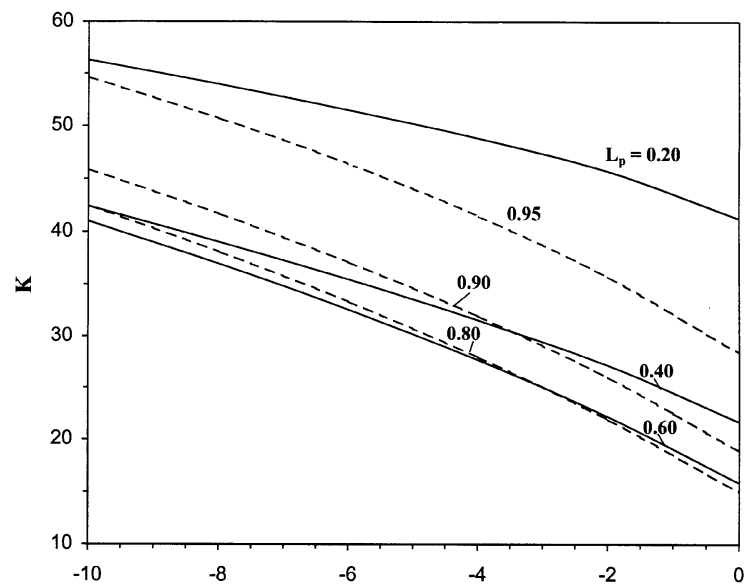

(a)

$\Theta$

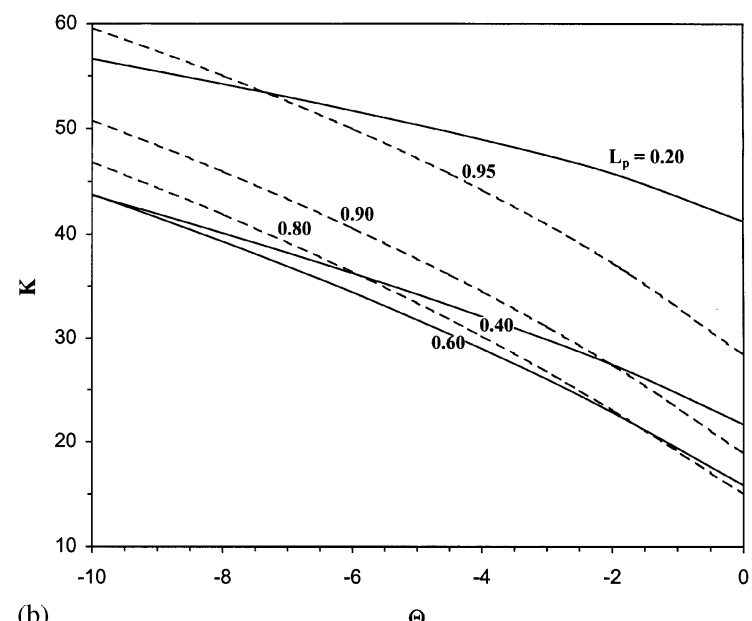

(b)

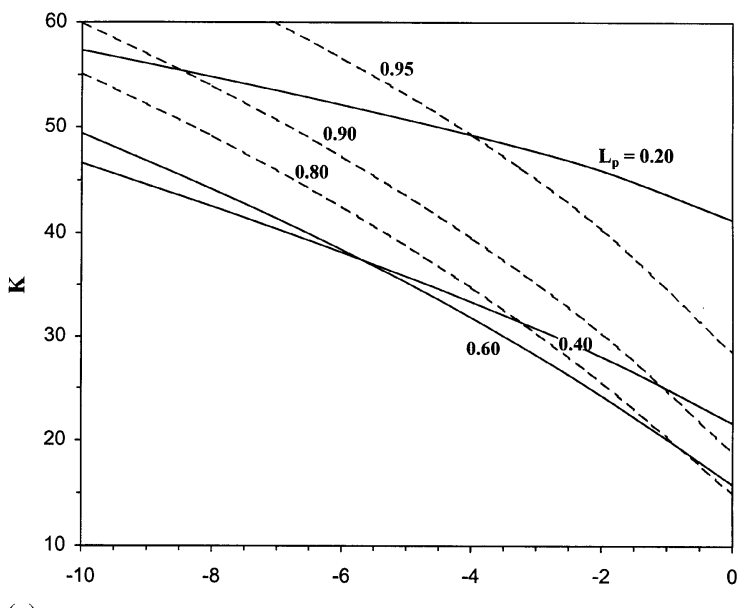

(c)

$\Theta$

Fig. 12. Normalized global stiffness, $K \equiv \mathscr{M}_{\lambda} / \psi$, versus normalized temperature, $\Theta$, for various patch lengths, $L_{\mathrm{p}}$; (a) $\alpha_{0}=\frac{1}{2}$, (b) $\alpha_{0}=1$, (c) $\alpha_{0}=2$. (Clamped-fixed supports).

properties. Two types of critical behavior were observed; bifurcation buckling and "asymptotic buckling". The occurrence and characteristics of such behavior were seen to be a function of the critical parameters. For the case of the edges being fixed from rotation, the characteristics of several configurations were investigated. In this case there were no issues of instability, ${ }^{11}$ but

${ }^{11}$ This differs from the behavior of similarly configured and supported structures during heating, for which a "sling-shot" instability was found to occur under appropriate circumstances $[1]$. a patch length yielding a maximum deflection was identified.

Finally, we remark that it is anticipated that symmetric patching (patching below as well as above the base plate) will generally negate transverse deflection for the loading scenarios considered, though the possibility of bifurcation buckling will be retained. Such a design, however, would be difficult to implement since it would involve patching interior surfaces as well as exterior surfaces of the base structure.

To close, it was seen that cooling of a patched structure may induce intricate and sometimes 

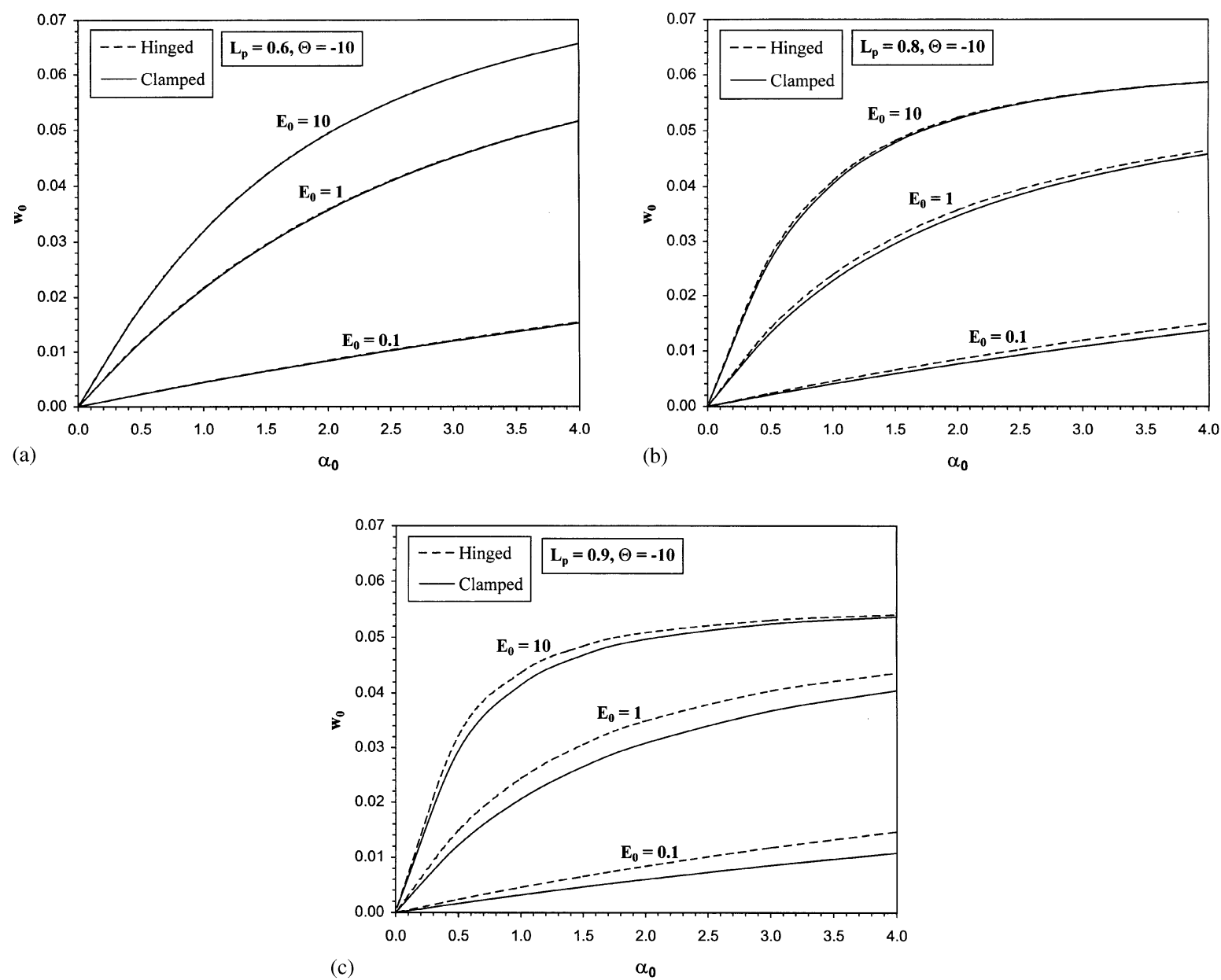

Fig. 13. Normalized transverse centerspan displacement, $w_{0}$, versus the ratio of coefficients of thermal expansion, $\alpha_{0}$, for hinged and clamped supports and various relative stiffnesses; (a) $L_{\mathrm{p}}=0.6$, (b) $L_{\mathrm{p}}=0.8$, (c) $L_{\mathrm{p}}=0.9$. $(\Theta=-10)$.

unexpected behavior, hence structures must be carefully designed, to achieve acceptable and required performance.

\section{Appendix A. Deformation-displacement relations}

Strain-displacement and curvature-displacement relations of the centerline of the base structure and patch, respectively

$$
\begin{aligned}
& e_{i}=u_{i}^{\prime}+\frac{1}{2} w_{l}^{\prime 2}, \quad x \in S_{i}(i=1,2), \\
& \kappa_{i}=w_{i}, \quad x \in S_{i}(i=1,2)
\end{aligned}
$$

$$
\begin{aligned}
& e_{\mathrm{p}}=u_{\mathrm{p}}^{\prime}+\frac{1}{2} w_{\mathrm{p}}^{\prime 2}, \quad x \in S_{1} \\
& \kappa_{\mathrm{p}}=w_{\mathrm{p}}, \quad x \in S_{1}
\end{aligned}
$$

where $h \ll 1$ and $h_{\mathrm{p}} \ll 1$ correspond to the normalized thickness of the base panel and patch, respectively.

In-plane displacements and membrane strains at the reference surface:

$$
\begin{aligned}
& u_{i}^{*}(x)=u_{i}(x)+\frac{1}{2} h w_{i}^{\prime} \quad(i=1,2), \\
& u_{\mathrm{p}}^{*}(s)=u_{\mathrm{p}}(x)-\frac{1}{2} h_{\mathrm{p}} w_{\mathrm{p}}^{\prime}, \\
& e_{i}^{*}(x)=e_{i}(x)+\frac{1}{2} h \kappa_{i} \quad(i=1,2), \\
& e_{\mathrm{p}}^{*}(x)=e_{\mathrm{p}}(x)-\frac{1}{2} h_{\mathrm{p}} \kappa_{\mathrm{p}} .
\end{aligned}
$$


(Superposed primes indicate total differentiation with respect to $x$.)

\section{Appendix B. Stiffnesses}

Normalized membrane and bending stiffness of base plate and patch:

$C=12 / h^{2}$,

$D=1$,

$C_{\mathrm{p}}=C E_{0} h_{0}$,

$D_{\mathrm{p}}=E_{0} h_{0}^{3}$,

where

$h_{0}=h_{\mathrm{p}} / h$.

Modulus ratio of patch to base panel:

$E_{0}=\frac{\bar{E}_{\mathrm{p}}}{\bar{E}} \quad$ (plane stress)

or

$E_{0}=\frac{\bar{E}_{\mathrm{p}} /\left(1-v_{\mathrm{p}}^{2}\right)}{\bar{E} /\left(1-v^{2}\right)} \quad($ plane strain $)$,

where $\bar{E}$ and $\bar{E}_{\mathrm{p}}$ correspond to the dimensional elastic moduli of the base panel and patch, respectively, and $v$ and $v_{\mathrm{p}}$ correspond to the associated Poisson's ratios.

Ratio of coefficients of thermal expansion:

$\alpha_{0} \equiv \alpha_{\mathrm{p}} / \alpha$,

where

$\alpha=\alpha^{0} \quad$ (plane stress)

and

$\alpha_{\mathrm{p}}=\alpha_{\mathrm{p}}^{0} \quad$ (plane stress)

or

$\alpha=(1+v) \alpha^{0} \quad$ (plane strain)

and

$\alpha_{\mathrm{p}}=\left(1+v_{\mathrm{p}}\right) \alpha_{\mathrm{p}}^{0} \quad$ (plane strain).
The non-dimensional coefficients of thermal expansion of the base structure and the patch, $\alpha^{0}$ and $\alpha_{p}^{0}$, respectively, are the products of the corresponding dimensional coefficients and reference temperature.

Normalized stiffnesses and thermal coefficients of patched segment of the composite structure:

$$
\begin{aligned}
& A^{*}=D+D_{\mathrm{p}}+\left(\frac{1}{2} h\right)^{2} C+\left(\frac{1}{2} h_{\mathrm{p}}\right)^{2} C_{\mathrm{p}}, \\
& B^{*}=\frac{1}{2} h_{\mathrm{p}} C_{\mathrm{p}}-\frac{1}{2} h C, \\
& C^{*}=C+C_{\mathrm{p}}, \\
& D^{*}=A^{*}-\rho^{*} B^{*}, \\
& \alpha^{*}=\alpha_{1}-\rho^{*} \beta^{*}, \\
& \beta^{*}=m^{*} / D^{*}, \\
& \rho^{*}=B^{*} / C^{*}, \\
& \mu^{*}=\frac{1}{2} h_{\mathrm{p}} C_{\mathrm{p}} \alpha_{\mathrm{p}}-\frac{1}{2} h C \alpha, \\
& n^{*}=C_{\mathrm{p}} \alpha_{\mathrm{p}}+C \alpha, \\
& m^{*}=\mu^{*}-\rho^{*} n^{*}, \\
& \alpha_{1}=n^{*} / C^{*} .
\end{aligned}
$$

\section{Appendix C. Normalized edge load}

The normalized edge load is given by

$T_{0}=\bar{T} \bar{L}^{2} / \bar{D}$,

where $\bar{T}$ is the dimensional in-plane load.

\section{References}

[1] A.M. Karlsson, W.J. Bottega, On thermal buckling of patched beam-plates, Int. J. Solids Struct. (1999) in press.

[2] G.L. Roderick, Prediction of cyclic growth of cracks and debonds on aluminum sheets reinforced with boron/epoxy, in: E.M. Lenoe, D.W. Oplinger, J.J. Burke (Eds.), Fibrous Composites in Structural Design, Plenum Press, New York, 1980, pp. 467-481.

[3] G.C. Sih, T.B. Hong, Integrity of edge-debonded patch on cracked panel, Theoret. Appl. Fract. Mech. 12 (1989) 121-143. 
[4] A.A. Baker, Repair efficiency in fatigue-cracked aluminum components reinforced with boron/epoxy patches, Fatigue Fract. Eng. Struct. 66 (1993) 753-765.

[5] W.J. Bottega, Separation failure in a class of bonded plates, Compos. Struct. 30 (1995) 253-269.

[6] W.J. Bottega, M.A. Loia, Edge debonding in patched cylindrical panels, Int. J. Solids Struct. 33 (1996) 3755-3777.

[7] W.J. Bottega, M.A. Loia, Axisymmetric edge debonding in patched plates, Int. J. Solids Struct. 34 (1997) 2255-2289.

[8] W.J. Bottega, A.M. Karlsson, On the detachment of steptapered doublers: Part 1 - Foundations, Int. J. Solids Struct. 36 (1999) 1597-1623.

[9] A.M. Karlsson, W.J. Bottega, The presence on edge contact and its influence on the debonding of patched panels, Int. J. Fract. 96 (1999) 383-406.

[10] A.M. Karlsson, W.J. Bottega, On the detachment of steptapered doublers: Part 2 - Evolution of pressure loaded structures, Int. J. Solids Struct. 36 (1999) 1621-1625.

[11] S. Naboulsi, S. Mall, Thermal effects on adhesively bonded composite repair of cracked aluminum panels, Theoret. Appl. Fract. Mech. 26 (1997) 1-12.

[12] M.R. Lena, J.C. Klug, C.T. Sun, Composite patches as reinforcements and crack arrestors in aircraft structures, J. Aircraft 35 (1998) 318-323.

[13] S. Timoshenko, Analysis of bi-metal thermostats, J. Opt. Soc. Am. Rev. Sci. Instr. 11 (1925) 233-255.

[14] A.M. Wahl, Analysis of valverde thermostat, J. Appl. Mech. 11 (1994) A183-A189.

[15] W.H. Wittrick, Stability of a bimetallic disk, Part I, Quart. J. Mech. Appl. Math. 6 (1953) 15-26.

[16] W.H. Wittrick, D.M. Myers, W.R. Blunden, Stability of a bimetallic disk, Part II, Quart. J. Mech. Appl. Math. 6 (1953) 26-31.

[17] N.N. Huang, T.R. Tauchert, Postbuckling response of antisymmetric angle-ply laminates to uniform temperature loading, Acta Mech. 72 (1988) 173-183.
[18] A. Hamamoto, M.W. Hyer, Non-linear temperaturecurvature relationships for unsymmetric graphiteepoxy laminates, Int. J. Solids Struct. 23 (1987) 919-935.

[19] R.C. Gauss, S.S. Antman, Large thermal buckling of nonuniform beams and plates, Int. J. Solids Struct. 20 (1984) 979-1000.

[20] T.R. Tauchert, Thermally induced flexure, buckling, and vibration of plates, Appl. Mech. Rev. 44 (1991) 347-360.

[21] A.K. Noor, W.S. Burton, Computational models for hightemperature multilayered composite plates and shells, Appl. Mech. Rev. 45 (1992) 419-446.

[22] A.K. Noor, J.M. Peters, Thermomechanical buckling of multilayered composite plates, J. Eng. Mech. 118 (1992) 351-366.

[23] A.K. Noor, J.H. Starnes Jr., J.M. Peters, Thermomechanical buckling and postbuckling of multilayered composite panels, Compos. Struct. 23 (1993) 233-251.

[24] L. Librescu, M.A. Souza, Post-buckling of geometrically imperfect shear-deformable flat panels under combined thermal and compressive edge loadings, J. Appl. Mech. 60 (1993) 526-533.

[25] G. Singh, G. Venkateswara Rao, N.G.R. Iyengar, Thermal post buckling behavior of rectangular antisymmetric cross-ply composite plates, Acta Mech. 98 (1993) 39-50.

[26] M.-L Dano, M.W. Hyer, Thermally-induced deformation behavior of unsymmetric laminates, Int. J. Solids Struct. 35 (1998) 2101-2120.

[27] W.-L. Yin, Thermomechanical buckling of delaminated composite laminates, Int. J. Solids Struct. 35 (1988) 2639-2653.

[28] G.J. Simitses, An Introduction to the Elastic Stability of Structures, Krieger Publishing Company, Malabar, Florida, 1986, pp. 125-127. 